\title{
Epiphyllous Liverworts of India: An Overview
}

\section{K. Singh ${ }^{1^{*}}$ and Devendra Singh ${ }^{2}$}

${ }^{1}$ Formerly Scientist G, Botanical Survey of India, 305D, Saraswati Apartment, Gomti Nagar Extension Sector 4, Lucknow - 226010 , India

${ }^{2}$ Botanical Survey of India, Central National Herbarium, Howrah -7111 03, India

Article history

Received: 30 March 2016

Accepted: 31 March 2016

Published: 02 May 2016

(c) Singh and Singh (2016)

Special Section: New Frontiers in Cryptogamic Botany

Section Editor

Afroz Alam

Publisher

Horizon e-Publishing Group

Corresponding Author

D. K. Singh

$\square_{\text {singh drdk@rediffmail.com }}$

\begin{abstract}
Taxonomy of the epiphyllous liverworts in India has been reviewed and their diversity and distribution has been discussed. A total of 160 species, one subspecies and two varieties of epiphyllous liverworts belonging to 23 genera in eight families have been recognized in Indian bryoflora, distributed only in eastern Himalaya and the north-east, Western Ghats, and the Andaman \& Nicobar Islands. Eastern Himalaya, including the north-east, with 133 taxa shows the maximum diversity of epiphylls, whereas Sikkim with 80 taxa is the richest amongst the States. Lejeuneaceae with 131 species belonging to 16 genera is the most prolific family of epiphyllous liverworts accounting for over 80 per cent of their total diversity in India, while Cololejeunea with 54 species is the most dominant genus. Twenty species are endemic to India, of which 11 are restricted to eastern Himalaya, three to Western Ghats, and one to Andaman \& Nicobar, while five species are common between eastern Himalaya and the Western Ghats.
\end{abstract}

\author{
Keywords \\ Epiphyllous liverworts; Diversity; Distribution; India
}

Singh, D. K., and D. Singh. 2016. Epiphyllous Liverworts of India: An Overview. Plant Science Today 3(2): 157-174. http://dx.doi.org/10.14719/pst.2016.3.2.228

\section{Introduction}

The vast geographical expanse of India and its extreme diversity of habitats have resulted in almost all types of ecosystems ranging from scrub to tropical evergreen rain forests, coastal mangroves to temperate and alpine vegetation in the country, with 10 distinct biogeographical zones, 27 biotic provinces and over 400 biomes. The biogeographically strategic location of the country at the confluence of three major global biogeographical realms, viz. Indo-Malayan, Eurasian and Afro-tropical has also contributed to the significant features of the Indian flora by the intermingling of the floristic elements from the surrounding countries. These biological attributes have contributed towards a rich biological diversity, including that of bryophytes, and have made India as one of the 17 mega biodiverse countries of the world ranking fourth in Asia and eleventh in the world (Mittermeier et al., 1997; Williams et al., 2001), accounting for about 11 per cent of the total global floristic resources. Based on distribution and floristic affinities of the liverworts, however, Pandé (1958) and Singh (1992, 1997) recognized eight bryogeographical regions in the country. The Indian liverworts and the hornworts, in the present state of our knowledge, represent about 12.40 per cent of their global diversity.

Bryophytes, because of their general lack of cuticle, absorb water and nutrients through the general surface of their plant body. Even in some mosses, where the conducting tissues are fairly evolved, and liverworts where rhizoids are abundantly present, they normally depend on surface absorption of water and nutrients. 


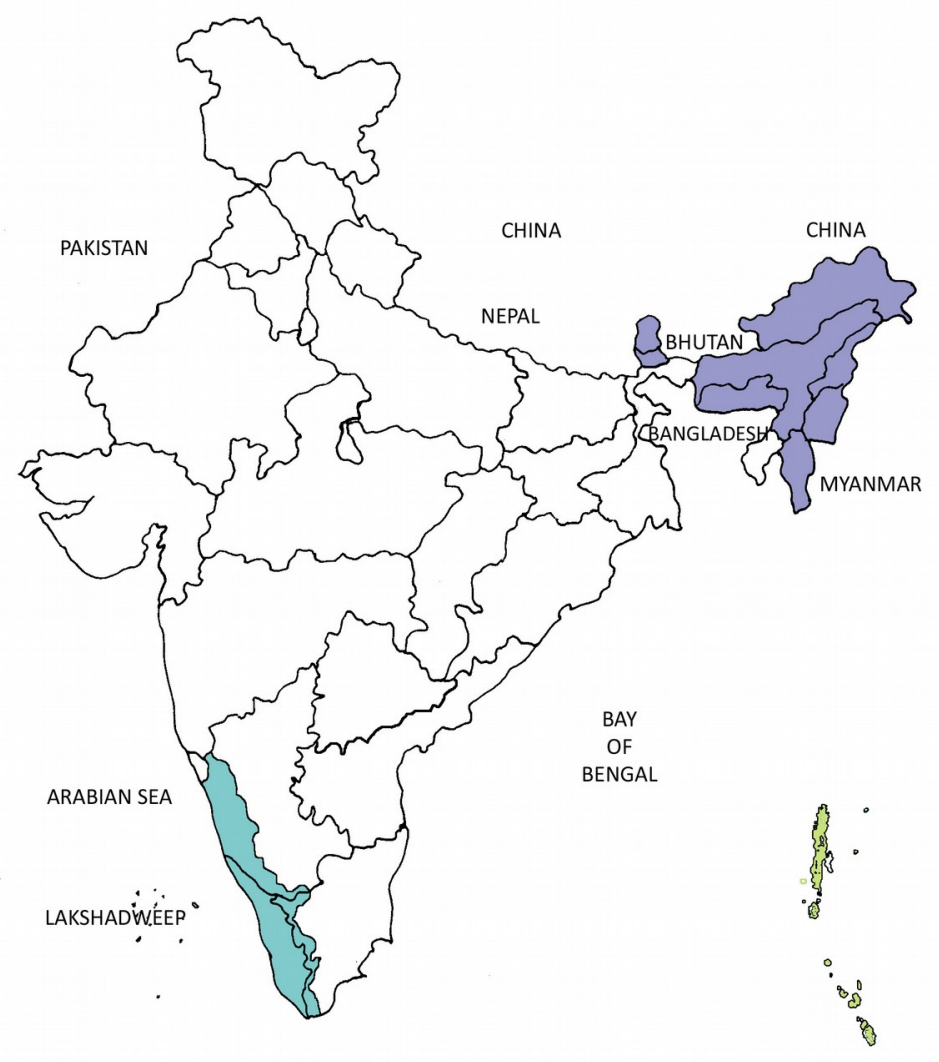

Fig. 1. Distribution of epiphyllous liverworts in India

Because of their poikilohydric nature, they grow on a variety of surfaces including abandoned articles, where usually no other plants will grow except algae, fungi and lichens. They may be terricolous, growing on soil; lignicolous, growing on decaying logs, leaf litter, etc; rupicolous or saxicolous, growing on bare rock surface; or corticolous, growing on the bark of vascular plants. In tropical and subtropical regions, which experience long periods of high humidity and where the condition of growth is very congenial, the bryophytes also grow as foliicolous or epiphyllous and form "phyllosphere" usually on the upper surface of leaves of vascular plants along with cyanobacteria, fungi and lichens. Interestingly, liverworts are the predominant constituents of the epiphyllous communities. In India eastern Himalaya, including the northeastern region, Western Ghats and the Andaman \& Nicobar Islands are the only bryogeographical regions of the country from where the epiphyllous liverworts have been recorded so far (Fig. 1).

\section{Taxonomy of Epiphyllous Liverworts in India}

The earliest report of epiphyllous liverwort from anywhere in the world dates back to later part of eighteenth century when Swartz (1788) described Jungermannia flava Sw. [=Lejeunea flava (Sw.) Nees], growing on fern fronds, from Jamaica. In India, Pandé and Misra (1943) for the first time described five epiphyllous species, viz. Leptocolea lanciloba (Steph.) A.Evans (=Cololejeunea lanciloba
Steph.), Rectolejeunea aloba (Sande Lac.) Steph. (=Lejeunea aloba Sande Lac.), Cololejeunea hispidissima (Steph.) Pandé, K.P.Srivast. \& S.Ahmad. [ $=C$. haskarliana (Lehm. \& Lindenb.) Schiffn.] from Western Ghats and Cololejeunea indica Pandé \& Misra and Leptolejeunea himalayensis Pandé \& Misra (=Lejeunea cocoes Mitt.) from eastern Himalaya. Later, Herzog (1950) described two new species viz. Strepsilejeunea ocellata Herzog (=Drepanolejeunea herzogii R.L.Zhu \& M.L.So) and Leptolejeunea latifolia Herzog from Darjeeling, while Kachroo (1951) reported epiphyllous Cololejeunea venusta (Sande Lac.) Schiffn. (Cololejeunea haskarliana) and Leptocolea lanciloba (=Cololejeunea lanciloba) from Assam. Few years later, Pandé et al. (1957) described Taeniolejeunea peraffinis (Schiffn.) Zwickel [=Cololejeunea appressa (A.Evans) Benedix], Taeniolejeunea pseudofloccosa (Horik.) Hatt. [=Cololejeunea pseudofloccosa (Horik.) Benedix], Leptolejeunea schiffneri Steph. $[=L$. elliptica (Lehm. \& Lindenb.) Schiffn.] and Drepanolejeunea foliicola Horik. from Western Ghats and the eastern Himalaya with notes on the distribution of another 23 epiphyllous taxa from the above two bryogeographical regions of the country and the Andaman \& Nicobar islands. Srivastava (1960) reported Riccardia levieri Schiffn. growing as epiphyllous on dicot leaves near Gersoppa falls in the Western Ghats of Karnataka. Lal (1977) reported Colura tenuicornis (A.Evans) Steph. from Darjeeling in eastern 
Himalaya, and later he (Lal, 1980) and Udar and Awasthi (1985) reported C. ari Steph. and C. acroloba (Mont.) Jovet-Ast respectively from the Andaman Islands. Udar and Srivastava (1983, 1985) described C. mizutaniana Udar \& G.Srivast. and C. kashyapii Udar \& G.Srivast. from Karnataka. Earlier, Udar and Awasthi $(1982,1984)$ reported epiphyllous Leptolejeunea foliicola Steph. from Meghalaya and Rhaphidolejeunea yunnanensis P.C.Chen [=Drepanolejeunea yunnanensis (P.C.Chen) Grolle \& R.L.Zhu] from Arunachal Pradesh, West Bengal and Karnataka, and Lal (1979) reported epiphyllous occurrence of Metzgeria nilgiriensis Udar \& S.C.Srivast. from Manipur. Udar and Kumar (1984) described four epiphyllous species of Radula Dumort. from India, and Udar et al. (1985) reported the foliicolous occurrence of Cololejeunea furcilobulata (Berrie \& E.W.Jones) R.M.Schust. from Karnataka. Whereas, Awasthi (1986) reported four epiphyllous species of genus Leptolejeunea (Spruce) Schiffn., and Srivastava and Srivastava (1989a, b) described six species of Cololejeunea from eastern Himalaya and the Western Ghats. Singh (1996) reported nine epiphyllous liverworts from Namdapha Tiger Reserve and National Park in Arunachal Pradesh belonging to five genera in two families.

Asthana and Srivastava (2003) described 26 epiphyllous species of Cololejeunea from India, whereas Zhu and Long (2003) reported four epiphyllous liverworts, Colura tenuicornis, Lejeunea flava, L. punctiformis Taylor [=Microlejeunea punctiformis (Taylor) Steph.] and Leptolejeunea latifolia from Darjeeling in eastern Himalaya. In a review of epiphyllous liverworts in India, Lal (2003) listed 38 species belonging to 13 genera of Lejeuneaceae and Metzgeria nilgiriensis of Metzgeriaceae. Singh et al. (2007) for the first time reported foliicolous existence of nine taxa of Metzgeria Raddi from Arunachal Pradesh, Sikkim and Himalayan West Bengal, while Singh and Singh (2008) reported foliicolous $M$. conjugata Lindb. from Sikkim. Daniels and Daniel (2004) reported the epiphyllous occurrence of Leptolejeunea balansae Steph. from Western Ghats, while Singh et al. (2006a) reported epiphyllous Cololejeunea desciscens Steph., Leptolejeunea balansae, L. epiphylla (Mitt.) Steph. and $L$. maculata (Mitt.) Schiffn. from Andaman Islands.

Asthana and Shukla (2009) described Drepanolejeunea fleischeri (Steph.) Grolle \& R.L.Zhu and D. pentadactyla (Mont.) Steph. for the first time in Indian liverwort flora from Karnataka, while Daniels and Daniel (2009) described Cololejeunea distalopapillata (E.W.Jones) R.M.Schust. and C. vidaliana Tixier from Tamil Nadu. Dey and Singh (2012) described 87 species and two varieties of epiphylls spreading across 19 genera and seven families from Arunachal Pradesh, Sikkim and Himalayan West Bengal. Singh et al. (2010a, b) reported 17 species belonging to five genera from Manipur, and five species belonging to genera Cololejeunea and
Leptolejeunea from Little Andaman respectively. Singh and Dey (2012) deiscibed Drepanolejeunea devendrae Sushil K. Singh \& M.Dey from Meghalaya, while Singh and Barbhuiya (2012) reported 31 species of epiphyllous liverworts from Assam. Daniels and Daniel (2013) reported eight species of epiphyllous liverworts from southern Western Ghats. Singh and Singh (2013) described Cololejeunea pluridentata P.C.Wu \& J.S.Lou for the first time in Indian liverwort flora and reported another five species of epiphyllous liverworts from Sikkim, while Singh and Barbhuiya (2013) recorded Cololejeunea chenii Tixier for the first time in India from Mizoram, and Singh et al. (2014) recorded Chielolejeunea osumiensis (S.Hatt.) Mizut. for the first time in India from Arunachal Pradesh and Sikkim. Singh and Dey (2014) reported Cololejeunea epiphylla G. Asthana \& A. Shukla and C. gottschei (Steph.) Mizut. from Mizoram, while Singh et al. (2015) and Kumar et al. (2015) reported Lejeunea kashyapii Dey et al., and Drepanolejeunea fleischeri and D. yunnanensis respectively from Meghalaya. Recently, Dey and Singh (2016a, b) described Cololejeunea andamansis M.Dey \& D.K.Singh and recorded C. pseudoschmidtii Tixier, C. equialbi Tixier, Colura pluridentata Ast and Drepanolejeunea thwaitesiana (Mitt.) Steph. var. thwaitesiana for the first time in Indian bryoflora from Andman \& Nicobar Islands. Whereas, Singh et al. (2016) recorded 26 epiphylls, including six species of the genus Radula Dumort. from Andman $\&$ Nicobar Islands.

\section{Synopsis of the Epiphyllous Liverworts in India}

The data presented here is based on the published information available to the authors. A careful analysis of all the published binomials of epiphyllous taxa in India revealed the occurrence of 160 species, one subspecies and two varieties belonging to 23 genera in eight families in three bryogeographical territories of the country, viz. Eastern Himalaya (including the north-east), Western Ghats and the Andaman \& Nicobar (Table 1). This accounts for 18.31 per cent of the total liverwort diversity of the country. However, no epiphyllous liverworts have so far been recorded from Tripura in the north-east, the Western Ghats of Goa, Dadra, Nagar \& Haveli and Maharashtra, and other bryogeographical regions of the country. Lejeuneaceae with 131 species belonging to 16 genera is the most prolific family of epiphyllous liverworts accounting for 80.36 per cent of the total taxa, followed distantly by Radulaceae and Metzgeriaceae with nine taxa each in genera Radula and Metzgeria respectively (5.5\%), Plagiochilaceae with six species (3.6\%), Frullaniaceae with four taxa (2.4\%), Lophocoleaceae with two species (1.2\%) and family Aneuraceae and Jubulaceae with one species each (0.61\%) (Table 1). Cololejeunea with 54 species is the largest genus of epiphylls in India, followed by Lejeunea (19), Drepanolejeunea (14), 
Leptolejeunea (11), Metzgeria and Radula (09 each), Cheilolejeunea and Colura (07 each), Plagiochila (06), Frullania, Lopholejeunea and Microlejeunea (04 each), Acrolejeunea (03) and Caudalejeunea and Heteroscyphus (02 each). On the other hand seven genera, viz. Acanthocoleus, Jubula, Lepidolejeunea, Metalejeunea, Ptychanthus, Riccardia and Tuyamaella are represented by a single species only.While all the seven species of the genus Colura hitherto known in Indian liverwort flora are epiphyllous, thus showing cent per cent epiphylly, 96.42 per cent of Indian species of the genus Cololejeuna, 91.66 per cent of Leptolejeunea, 77.77 per cent of Drepanolejeunea, 42.85 per cent of Metzgeria, 38 per cent of Lejeunea, etc. occur as epiphyllous as well.

Table 1. Conspectus of epiphyllous liverworts in India

\begin{tabular}{|c|c|c|c|}
\hline Order & Family & Genus & Species \\
\hline \multirow[t]{3}{*}{ Metzgeriales } & Aneuraceae & Riccardia & 1 \\
\hline & Metzgeriaceae & Metzgeria & $8(1)$ \\
\hline & Radulaceae & Radula & 9 \\
\hline \multirow[t]{18}{*}{ Porellales } & Frullaniaceae & Frullania & $2(2)$ \\
\hline & Jubulaceae & Jubula & 1 \\
\hline & \multirow{16}{*}{ Lejeuneaceae } & Acanthocoleus & 1 \\
\hline & & Acrolejeunea & 3 \\
\hline & & Archilejeunea & 1 \\
\hline & & Caudalejeunea & 2 \\
\hline & & Cheilolejeunea & 7 \\
\hline & & Cololejeunea & 54 \\
\hline & & Colura & 7 \\
\hline & & Drepanolejeunea & 14 \\
\hline & & Lejeunea & 19 \\
\hline & & Lepidolejeunea & 1 \\
\hline & & Leptolejeunea & 11 \\
\hline & & Lopholejeunea & 4 \\
\hline & & Metalejeunea & 1 \\
\hline & & Microlejeunea & 4 \\
\hline & & Ptychanthus & 1 \\
\hline & & Tuyamaella & 1 \\
\hline \multirow[t]{2}{*}{ Jungermanniales } & Lophocoleaceae & Heteroscyphus & 2 \\
\hline & Plagiochilaceae & Plagiochila & 6 \\
\hline Total & 8 & 23 & 163 \\
\hline
\end{tabular}

The figures in parenthesis represent the infraspecific taxa.

The taxa at the rank of genus and above are treated as per the classification proposed by Crandall-Stotler et al. (2009) with some modifications based on Gradstein (2013) and Wang et al. (2014). The genera, and the species within each genus, are arranged alphabetically. Currently accepted binomials are provided in boldface, while the basionyms, if any, and the synonyms are in italic font. The name of a taxon is followed by nomenclatural citation, state-wise distrubution with the names of the states given alphabetically in acronym, and the relevant literature reference. The names of the authors of plant names have been abbreviated according to Brummitt and Powell (1992), while the journals have been abbreviated as per Bridson et al. (2004a, b).

Acronyms used: AN: Andaman \& Nicobar; AR: Arunachal Pradesh; AS: Assam; EH: Eastern Himalaya; KA: Karnataka; KL: Kerala; ML: Meghalaya; MN: Manipur; MZ: Mizoram; NL: Nagaland; SK: Sikkim; TN: Tamil Nadu; WG: Western Ghats; WB: West Bengal.

\section{Enumeration}

Acanthocoleus R.M.Schust. (Lejeuneaceae)

Acanthocoleus gilvus (Gottsche) Kruijt, Bryophyt. Biblioth. 36: 79. 1988. ML, SK, WB (Lal, 2003; Dey and Singh, 2012).

Acrolejeunea (Spruce) Schiffn. (Lejeuneaceae)

- Acrolejeunea infuscata (Mitt.) J.Wang bis \& Gradst. in Wang et al., Bry. Div. Evo. 36: 38. 2014. SK (Dey and Singh, 2012 as Trocholejeunea infuscata).

- Acrolejeunea pusilla (Steph.) Grolle \& Gradst., J. Hattori Bot. Lab. 38: 332. 1974. SK (Singh and Singh, 2013).

- Acrolejeunea sandvicensis (Gottsche) J.Wang bis \& Gradst., in Wang et al., Bry. Div. Evo. 36: 39. 2014. AR, SK (Dey et al., 2009; Dey and Singh, 2012, both as Trocholejeunea sandvicensis).

- Aneura levieri Schiffn. ex Steph., Bull. Herb. Boissier 7: 751. 1899 \& Sp. Hepat. 1: 261. 1899 = Riccardia levieri

- Aphanolejeunea diaphana (A.Evans) R.M.Schust., Hep. Anthoc. N. Amer. 4: 1294. 1980. nom. illeg., non Aphanolejeunea diaphana Herzog. = Cololejeunea diaphana

- Aphanolejeunea grossepapillosa Horik., J. Sci. Hiroshima Univ., Ser. B, Div. 2, Bot. 1: 92. 1932 = Cololejeunea grossepapillosa

- Aphanolejeunea truncatifolia Horik., J. Sci. Hiroshima Univ., Ser. B, Div. 2, Bot. 2: 284. 1934 = Cololejeunea grossepapillosa

Archilejeunea (Spruce) Schiffn. (Lejeuneaceae)

- Archilejeunea apiculifolia Steph., Sp. Hepat. 6: 558. 1924 = Archilejeunea planiuscula

- Archilejeunea apiculifolia Steph. var. dentifolia U.S. Awasthi \& S.C. Srivast. Geophytology 18: 207. $1988=$ Archilejeunea planiuscula

- Archilejeunea planiuscula (Mitt.) Steph., Sp. Hepat. 4: 731. 1911. AS (Singh and Barbhuiya, 2012). 
- Archilejeunea pusilla Steph., Sp. Hepat. 4: 731. 1911 = Acrolejeunea pusilla

- Brachiolejeunea andamana Steph., Sp. Hepat. 5: 130. 1912 = Ptychanthus striatus

- Brachiolejeunea infuscata (Mitt.) R.M.Schust., Beih. Nova Hedwigia 9: 105. $1963=$ Acrolejeunea infuscata

- Brachiolejeunea levieri Steph., Sp. Hepat. 5: 134. 1912 = Acrolejeunea infuscata

- Brachiolejeunea sandvicensis (Gottsche) A.Evans, Trans. Connecticut Acad. Arts 10: 419. $1900=$ Acrolejeunea sandvicensis

- Bryopteris nepalensis Steph., Sp. Hepat. 5: 9. 1912 $=$ Ptychanthus striatus

Caudalejeunea (Steph.) Schiffn. (Lejeuneaceae)

- Caudalejeunea lehmanniana (Gottsche) A.Evans, Bull. Torrey Bot. Club 34: 544. 1907. AR (Dey and Singh, 2009; Dey and Singh, 2012).

- Caudalejeunea pluriplicata Udar, U.S. Awasthi \& Shaheen, Bryologist 85: 329. $1982=$ Caudalejeunea reniloba

- Caudalejeunea reniloba (Gottsche) Steph., Sp. Hepat. 5: 16. 1912. AN, AS, KA (Lal, 2003; Singh and Barbhuiya, 2012; Singh et al., 2016 as $C$. recurvistipula).

Cheilolejeunea (Spruce) Schiffn. (Lejeuneaceae)

- Cheilolejeunea imbricata (Nees) S. Hatt., Misc. Bryol. Lichenol. 1(14): 1. $1957=$ Cheilolejeunea trapezia

- Cheilolejeunea intertexta (Lindenb.) Steph.,Hedwigia 29: 85. 1890. AN (Lal, 2003).

- Cheilolejeunea katschalliana Steph., Hedwigia 34: 242. 1895 = Cheilolejeunea intertexta

- Cheilolejeunea kurzii Steph., Bot. Gaz. 15: 284. 1890 = Cheilolejeunea intertexta

- Cheilolejeunea laeviuscula (Mitt.) Steph., Sp. Hepat. 5: 668. 1914. AS, SK (Dey and Singh, 2012; Singh and Barbhuiya, 2012 as Cheilolejeunea longiloba).

- Cheilolejeunea longiloba (G.Hoffm.) J.J. Engel \& B.C.Tan, J. Hattori Bot. Lab. 60. 294. $1986=$ Cheilolejeunea laeviuscula

- Cheilolejeunea osumiensis (S.Hatt.) Mizut., Misc. Bryol. Lichenol. 8: 148. 1980. AR, SK (Singh et al., 2014).

- Cheilolejeunea serpentina (Mitt.) Mizut., J. Hattori Bot. Lab. 26: 171. 1963. AS (Singh and Barbhuiya, 2012).

- Cheilolejeunea subopaca (Mitt.) Mizut., J. Hattori Bot. Lab. 26: 183. 1963. KL (Manju et al., 2012).

- Cheilolejeunea trapezia (Nees) Kachroo \& R.M.Schust., J. Linn. Soc. London, Bot. 56: 509. 1961. AR, SK, TN, WB (Lal, 2003 as $C$. ceylanica; Dey and Singh, 2012).
- Cheilolejeunea trapezia (Nees) Mizut., J. Hattori Bot. Lab. 24: 282. 1961 = Cheilolejeunea trapezia

- Cheilolejeune trifaria (Reinw., Blume \& Nees) Mizut., J. Hattori Bot. Lab. 27: 132. 1964. AS (Singh and Barbhuiya, 2012).

- Chiloscyphus communis Steph., Bull. Herb. Boissier, Ser. 2, 7: 839. 1907 \& Sp. Hepat. 3: 211. 1907 = Heteroscyphus bescherellei

- Chiloscyphus gammianus Steph., Bull. Herb. Boissier, Ser. 2, 7: 845. 1907 \& Sp. Hepat. 3: 217. $1907=$ Heteroscyphus hyalinus

Cololejeunea (Spruce) Schiffn. (Lejeuneaceae)

- Cololejeunea acinacifolia (Steph.) Kitag., J. Hattori Bot. Lab. 36: 451. 1972 = Cololejeunea longifolia

- Cololejeunea aequabilis (Sande Lac.) Schiffn., Consp. Hep. Archip. Indici 242. 1898. AR (Dey and Singh, 2011a, 2012).

- Cololejeunea andamanensis M.Dey \& D.K.Singh, Cryptog. Bryol. 37(2): 150. 2016. AN (Dey and Singh, 2016b).

- Cololejeunea appresa (A.Evans) Benedix, Feddes Repert. Beih. 134: 31.1953. AS, KA, TN, WB (Asthana and Srivastava, 2003; Verma and Srivastava, 2008; Singh and Barbhuiya, 2012).

- Cololejeunea bhutanica Grolle \& Mizut., J. Bryol. 15: 281. 1988. SK (Dey and Singh, 2011a, 2012).

- Cololejeunea bolombensis (Steph.) Vanden Berghen, Bull. Jard. Bot. Belg. 42: 475. 1975 = Cololejeunea lanciloba

- Cololejeunea cardiocarpa (Mont.) A.Evans, Hedwigia 29. 91. 1890. KL, TN (Asthana and Srivastava, 2003; Verma and Srivastava, 2008).

Cololejeunea ceratilobula (P.C.Chen) R.M.Schust., Beih. Nova Hedwigia 9: 179. 1963 AN, AR, SK (Dey and Singh, 2012; Singh and Singh, 2013; Singh et al., 2016).

- Cololejeunea ceylanica Onr., Acta Bot. Acad. Sci. Hung. 25: 107. 1979. AR (Asthana and Srivastava, 2003; Dey and Singh, 2012).

- Cololejeunea chenii Tixier, Bryphot. Biblioth. 27. 219. 1985. MZ, SK (Singh and Barbhuiya, 2013; Singh and Singh, 2013).

- Cololejeunea denticulata (Horik.) S.Hatt., Bull. Tokyo Sci. Mus. 11: 99. 1944. AS (Singh and Barbhuiya, 2012).

- Cololejeunea dentifolia Udar \& G. Srivast., J. Bryol. 12: 229. 1982 = Cololejeunea gottschei

- Cololejeunea desciscens Steph., Hedwigia 24. 248. 1895. AN, AS (Singh et al., 2006a, 2010b; Singh and Barbhuiya, 2012).

- Cololejeunea diaphana A.Evans, Bull. Torrey Bot. Club 32: 184. 1905. AR, SK, WB (Asthana and Srivastava, 2003; Dey and Singh, 2012, both as $C$. truncatifolia). 
- Cololejeunea distalopapillata (E.W.Jones) R.M.Schust., Beih. Nova Hedwigia 9: 173. 1963. TN (Daniels and Daniel, 2009, 2013).

- Cololejeunea dozyana (Sande Lac.) Schiffn., Consp. Hepat. Arch. Ind. 242. 1898. SK (Dey and Singh, 2011a, 2012).

- Cololejeunea epiphylla G. Asthana \& A. Shukla, Cryptog. Bryol. 31: 218. 2010. KA, MZ (Asthana and Shukla, 2010; Singh and Dey, 2014).

- Cololejeunea equialbi Tixier, Ann. Fasc. Sci. Univ. Phnom Penh. 3: 178. 1970. AN (Singh et al., 2016; Dey and Singh, 2016a).

- Cololejeunea floccosa (Lehm. \& Lindenb.) Schiffn., Consp. Hepat. Arch. Ind. 243. 1898. AN, AR, SK (Dey and Singh, 2012; Singh et al., 2016).

- Cololejeunea foliicola S.C.Srivast. \& G. Srivast., Proc. Indian Acad. Sci., Pl. Sci. 99: 86. 1989. AS, KA, KL (Asthana and Srivastava, 2003; Manju et al., 2012; Singh and Barbhuiya, 2012).

- Cololejeunea furcilobulata (Berrie \& E.W.Jones) R.M.Schust., Beih. Nova Hedwigia 9: 178. 1963. KA (Asthana and Srivastava, 2003)

- Cololejeunea goebelii (Gottsche ex K.I. Goebel) Schiffn., Consp. Hepat. Arch. Ind. 244. $1898=$ Cololejeunea trichomanis

- Cololejeunea goebelii (Gottsche ex K.I. Goebel) Schiffn. var. acrotremae (Schiffn.) Schiffn., Consp. Hepat. Arch. Ind. 244. $1898=$ Cololejeunea jelinekii

- Cololejeunea gottschei (Steph.) Mizut., J. Hattori Bot. Lab. 28: 117. 1965. AN, KA, MZ (Udar \& Srivastava, 1982; Lal, 2003, both as as $C$. dentifolia; Asthana and Srivastava, 2003; Singh et al., 2010b; Singh and Dey, 2014).

- Cololejeunea grossepapillosa (Horik.) Pócs, Polish Bot. J. 54 (1): 5. 2009. AN, SK (Dey and Singh, 2012; Singh et al., 2016).

- Cololejeunea haskarliana (Lehm. \& Lindenb.) Schiffn., Consp. Hepat. Arch. Ind. 244. 1898. AN, AR, KA, MN, SK, TN (Pande \& Misra, 1943 as C. hispidissima; Lal, 2003; Singh et al., 2010a; Dey and Singh, 2012; Singh et al., 2016).

- Cololejeunea himalayensis (Pande \& R.N.Misra) R.M.Schust., Beih. Nova Hedwigia 9: 177. 1963 = Cololejeunea latilobula

- Cololejeunea hispidissima (Steph.), Pande, K.P.Srivast. \& S.Ahmad., J. Indian Bot. Soc. 36: 343.1957 = Cololejeunea haskarliana

- Cololejeunea hyalina G.Asthana \& S.C.Srivast., Bryophyt. Biblioth. 60: 25. 2003. KA (Asthana and Srivastava, 2003).

- Cololejeunea hyalina Parihar, Lal \& Katiyar, Hepat. Anth. Indian Ann. Checkl. Allahabad 19. 1994, nom. illeg. = Cololejeunea hyalina
- Cololejeunea indica Pandé \& R.N. Misra, J. Indian Bot. Soc. 22: 166. 1943. MZ, WB (Pande \& Misra, 1943; Singh \& Pócs, 2016).

- Cololejeunea inflata Steph., Hedwigia 34: 249. 1895. AS (Singh and Barbhuiya, 2012).

- Cololejeunea jelinekii Steph., Hedwigia 34: 249. 1895. AN, AR (Asthana and Srivastava, 2003; Lal, 2003 as Cololejeunea acrotremae; Das and Singh, 2009).

- Cololejeunea karnatakensis G.Asthana \& S.C.Srivast., Bryopht. Biblioth. 60: 26. 2003. KA (Asthana and Srivastava, 2003).

- Cololejeunea karnatakensis Parihar, Lal \& Katiyar, Hepat. Anth. India Ann. Checkl. Allahabad 19. 1994, nom. illeg.= Cololejeunea karnatakensis

- Cololejeunea kashyapii Udar \& G. Srivast., Geophytology 15: 64. 1985. AS, KA (Asthana and Srivastava, 2003; Lal, 2003; Singh and Barbhuiya, 2012).

- Cololejeunea lanciloba Steph., Hedwigia 34: 250. 1895. AS, KA, MN, SK, TN (Pande \& Misra, 1943 as Leptocolea lanciloba; Singh et al., 2010a; Dey and Singh, 2012; Singh and Barbhuiya, 2012; Daniels and Daniel, 2013).

- Cololejeunea latilobula (Herzog) Tixier, Bryophyt. Biblioth. 27:156. 1985. AR, ML, MN, SK, WB (Asthana and Srivastava, 2003; Singh et al., 2010a; Dey and Singh, 2012; Singh and Barbhuiya, 2012).

- Cololejeunea longiana Grolle \& Mizut., J. Bryol. 15: 284. 1988. SK, WB (Dey and Singh, 2008a, 2012).

- Cololejeunea longifolia (Mitt.) Benedix ex Mizut., J. Hattori Bot. Lab. 26: 184. 1963. AN, AR, KA, MN, SK, WB (Asthana and Srivastava, 2003; Lal, 2003; Singh et al., 2010a; Dey and Singh, 2012).

- Cololejeunea macounii (Spruce ex Underw.) A.Evans, Torrey Bot. Club 8: 171. 1902. SK (Dey et al., 2010; Dey and Singh, 2012).

- Cololejeunea madothecoides (Steph.) Benedix, Feddes Repert. Spec. Nov. Regni Veg. Beih.134: 81. 1953. KL (Manju et al., 2012).

- Cololejeunea minutissima (Sm.) Schiffn., Engler \& Prantl, Nat. Pflanzenfam. l (3): 122. 1895. TN (Daniels and Daniel, 2013).

- Cololejeunea mizutaniana Udar \& G. Srivast., Misc. Bryol. Lichenol. 9: 137. 1983. AS (Singh and Barbhuiya, 2012).

- Cololejeunea nilgiriensis G. Asthana \& S.C. Srivast., Bryophyt. Biblioth. 60: 27. 2003. AR, SK (Das and Singh, 2009; Singh and Singh, 2013).

- Cololejeunea ocellata (Horik.) Benedix, Feddes Repert. Spec. Nov. Regni Veg. Beih. 134: 38. 1953. SK (Dey et al., 2010; Dey and Singh, 2012). 
- Cololejeunea pandei Udar \& G. Srivast., J. Bryol. 12: 227.1982 = Cololejeunea trichomanis

- Cololejeunea peraffinis (Schiffn.) Schiffn., Consp. Hepat. Arch. Ind. 245. 1898. KA, WB (Pande et al., 1957; Lal, 2003).

- Cololejeunea planissima (Mitt.) Abeyw., Ceylon J. Sci., Bio. Sci. 2: 73. 1959. AR (Dey and Singh, 2012).

- Cololejeunea pluridentata P.C.Wu \& J.S.Lou, Acta Phytotax. Sin. 16: 105. 1978. SK (Singh and Singh, 2013).

- Cololejeunea pseudofloccosa (Horik.) Benedix, Feddes Repert. Beih. 134: 36. 1953. AR, MN, SK, TN, WB (Lal, 2003; Singh et al., 2010; Dey and Singh, 2012; Singh and Singh, 2013).

- Cololejeunea pseudoplagiophylla P.C.Wu \& J.S.Lou, Acta Phytotax. Sin. 16: 105. 1978. AR (Singh, 1996; Asthana and Srivastava, 2003; Dey and Singh, 2012).

- Cololejeunea pseudoschmidtii Tixier, Gard. Bull. Singapore 26: 145. 1972. AN (Dey and Singh, 2016b).

- Cololejeunea raduliloba Steph., Hedwigia 34: 251. 1895. KL (Manju et al., 2012).

- Cololejeunea serrulata Steph., Hedwigia 34. 252. 1895. AR, SK, WB (Dey and Singh, 2011a, 2012; Singh and Singh, 2013).

- Cololejeunea siangensis G.Asthana \& S.C.Srivast., Bryophyt. Biblioth. 60: 57. 2003. AR, AS (Asthana and Srivastava, 2003; Lal, 2003; Dey and Singh, 2012; Singh and Barbhuiya, 2012).

- Cololejeunea siangensis Parihar, Lal \& Katiyar, Hepat. Anth. India Ann. Checkl. Allahabad 19. 1994, nom. illeg. = Cololejeunea siangensis

- Cololejeunea sigmoidea Jovet-Ast. \& Tixier, Rev. Bryol. Lichénol. 31: 27. 1962. KA, TN (Asthana and Srivastava, 2003; Sreebha et al., 2015).

- Cololejeunea spinosa (Horik.) Pandé \& Misra, J. Indian Bot. Soc. 22: 166. 1943. AR, SK, TN, WB (Singh, 1996; Asthana and Srivastava, 2003; Lal, 2003; Dey and Singh, 2012; Singh and Singh, 2013; Daniels and Daniel, 2013).

- Cololejeunea subocelloides Mizut., J. Hattori Bot.Lab. 57: 163. 1984. SK (Singh, 2016).

- Cololejeunea tixieriana M.Dey, D. Singh \& D.K.Singh, Taiwania 53: 258. 2008. AR, SK, WB (Dey et al., 2008a; Dey and Singh, 2012).

- Cololejeunea trianguliloba (Steph.) Pande, K.P. Srivast. \& S. Ahmad, J. Indian Bot. Soc. 36: 343. $1957=$ Cololejeunea planissima

- Cololejeunea trichomanis (Gottsche) Steph., Hedwigia 34: 252. 1895. AR, AS, KA, MN, SK, WB (Lal, 2003 as C. goebelii; Singh et al., 2010a; Dey and Singh, 2012; Singh and Barbhuiya, 2012; Singh and Singh, 2013).
- Cololejeunea truncatifolia (Horik.) Mizut., J. Hattori Bot. Lab. 24: 282. $1961=$ Cololejeunea diaphana

- Cololejeunea udarii G.Asthana \& S.C.Srivast., Bryophyt. Biblioth. 60: 40. 2003. AR, KA, KL, MN, TN (Asthana and Srivastava, 2003; Verma and Srivastava, 2008; Manju et al., 2012; Singh and Barbhuiya, 2012).

- Cololejeuena venusta (Sande Lac.) Schiffn., Hepat. (Engl.-Prantl) 122. $1895=$ Cololejeunea haskarliana

- Cololejeunea vidaliana Tixier, Nat. Hist. Bull. Siam Soc. 24: 444. 1973. TN (Daniels and Daniel, 2009, 2013)

- Cololejeunea yipii R.L.Zhu, Nova Hedwigia 121: 346. 2001. AR (Dey and Singh, 2007, 2012).

Colura (Dumort.) Dumort. (Lejeuneaceae)

- Colura acroloba (Mont.) Jovet-Ast, Rev. Bryol. Lichénol. 22: 297. 1954 "1953". AN (Udar \& Awasthi, 1985; Lal, 2003).

- Colura acutifolia Jovet-Ast, Rev. Bryol. Lichénol. 22: 281.1953 = Colura conica

- Colura ari (Steph.) Steph., Sp. Hepat. 5: 936. 1916. AN, AS (Lal, 2003; Singh and Barbhuiya, 2012).

- Colura calyptrifolia (Hook.) Dumort., Recueil Observ. Jungerm. 12. 1835. AR, WB (Lal, 1977, 2003; Singh, 1996).

- Colura conica (Sande Lac.) K.I.Goebel, Ann. Jard. Bot. Buitenzorg 39: 3.1928. AR (Lal, 2003 as C. acutifolia).

- Colura leratii (Steph.) Steph., Sp. Hepat. 5: 939. 1916. KA (Asthana and Shukla, 2010).

- Colura pluridentata Ast, Rev. Bryol. Lichénol. 22: 265. 1954 "1953". AN (Dey and Singh, 2016a).

- Colura tenuicornis (A.Evans) Steph., Sp. Hepat. 5: 942. 1916. AR, SK, WB (Dey and Singh, 2012).

- Colurolejeunea acroloba Mont. ex Steph., Hedwigia 29: 97.1890 = Colura acroloba

- Colurolejeunea ari Steph., Hedwigia 35: 73. 1896 = Colura ari

- Colurolejeunea conica (Sande Lac.) Schiffn., Consp. Hepat. Arch. Ind. 258. 1898 = Colura conica

- Colurolejeunea leratii Steph., Rev. Bryol. 35: 30. $1908=$ Colura leratii

- Dicranolejeunea gilva (Gottsche) Steph., Hedwigia 35: 77. 1896 = Acanthocoleus gilvus

- Dicranolejeunea sikkimensis Steph., Sp. Hepat. 5: 170. 1912 = Acanthocoleus gilvus

Drepanolejeunea (Spruce) Schiffn. (Lejeuneaceae)

- Drepanolejeunea angustifolia (Mitt.) Grolle, J. Jap. Bot. 40: 206. 1965. SK, WB (Dey and Singh, 2012; Dey et al., 2013). 
- Drepanolejeunea apiculata Horik., J. Sci. Hiroshima Univ., Ser. B, Div. 2, Bot. 2: 266. 1934 = Leptolejeunea apiculata

- Drepanolejeunea devendrae Sushil K. Singh \& M. Dey, Nelumbo 54: 20. 2012. ML (Singh and Dey, 2012).

- Drepanolejeunea erecta (Steph.) Mizut., J. Hattori Bot. Lab. 40: 442. 1976. AR, MN, SK, WB (Singh et al., 2010a; Dey and Singh, 2012; Dey et al., 2013; Singh and Singh, 2013).

- Drepanolejeunea fleischeri (Steph.) Grolle \& R.L.Zhu, Nova Hedwigia 70: 379. 2000. AR, KA, ML, SK, WB (Asthana and Shukla, 2009; Dey and Singh, 2010a, 2012; Dey et al., 2013; Kumar et al., 2015).

- Drepanolejeunea foliicola Horik., J. Sci. Hiroshima Univ., Ser. B, Div. 2, Bot. 1: 85. 1932. WB (Lal, 2003; Dey et al., 2013).

- Drepanolejeunea herzogii R.L.Zhu \& M.L.So, Beih. Nova Hedwigia 121: 181. 2001. AR, MN, SK, WB (Singh et al., 2010a; Dey and Singh, 2012; Singh and Barbhuiya, 2012; Dey et al., 2013).

- Drepanolejeunea laciniata Qiong He \& R.L.Zhu, Cryptog. Bryol. 33: 292. SK (Singh, 2016).

- Drepanolejeunea longifolia A.P. Singh \& V.Nath, Hepat. Khasi Jaintia Hills: E. Himal. 248. 2007 \& Bryologist 111: 477. $2008=$ Drepanolejeunea angustifolia

- Drepanolejeunea longii Grolle \& R.L.Zhu, Ann. Bot. Fenn. 36: 115. 1999. SK (Dey and Singh, 2012; Singh and Singh, 2013; Dey et al., 2013).

- Drepanolejeunea monophthalma (Herzog) Mizut., J. Hattori Bot. Lab. 29: 291. $1966=$ Drepanolejeunea erecta

- Drepanolejeunea ocellata (Herzog) R.L.Zhu \& M.L.So, Beih. Nova Hedwigia 121: 182. 2001 (non Drepanolejeunea ocellata Steph., Sp. Hepat. 5: 349. 1913 (fide Zhu and So, 2001) = Drepanolejeunea herzogii

- Drepanolejeunea pentadactyla (Mont.) Steph., Sp. Hepat. 5: 357. 1913. AN, KA (Asthana and Shukla, 2009; Singh et al., 2016).

- Drepanolejeunea siamensis (Bischl.) Grolle \& R.L.Zhu, Nova Hedwigia 70: 393. 2000. SK (Dey and Singh, 2012; Dey et al., 2013).

- Drepanolejeunea sikkimensis (Udar \& U.S. Awasthi) Grolle, J. Hattori Bot. Lab. 55: 503. 1984 = Leptolejeunea sikkimensis

- Drepanolejeunea tenuis (Reinw., Blume \& Nees) Schiffn., Consp. Hepat. Arch. Ind. 280. $1898=$ Drepanolejeunea angustifolia

- Drepanolejeunea ternatensis (Gottsche) Steph., Hedwigia 28: 169. 1889. TN (Lal, 2003; Dey et al., 2013).

- Drepanolejeunea ternatensis (Gottsche) Steph. var. lancispina Herzog, Ann. Bryol. 12: 119. $1939=$ Drepanolejeunea ternatensis
- Drepanolejeunea thwaitesiana (Mitt.) Steph. Sp. Hepat. 5: 350. 1913.var. thwaitesiana. AN (Singh et al., 2016; Dey and Singh, 2016a).

- Drepanolejeunea tibetana (P.C.Wu \& J.S.Lou) Grolle \& R.L.Zhu, Nova Hedwigia 70: 386. 2000. AR (Dey and Singh, 2012; Dey et al., 2013).

- Drepanolejeunea yunnanensis (P.C.Chen) Grolle \& R.L.Zhu, Nova Hedwigia 70: 388. 2000. AR, AS, KA, ML, MN, TN, WB (Udar and Awasthi, 1984; Singh, 1996 as Rhaphidolejeunea yunnanensis; Singh et al., 2010a; Dey and Singh, 2012; Singh and Barbhuiya, 2012; Dey et al., 2013; Verma et al., 2013; Kumar et al., 2015).

- Eulejeunea aloba (Sande Lac.) Schiffn., Consp. Hepat. Arch. Ind. 247. $1898=$ Lejeunea aloba

- Euosmolejeunea brunnea Steph., Sp. Hepat. 6: 412. 1923 (syn. fide Mizutani, 1972) = Cheilolejeunea intertexta

- Euosmolejeunea osumiensis S.Hatt., Bull. Tokyo Sci. Mus. 11: 105. 1944 = Cheilolejeunea osumiensis

- Euosmolejeunea papillata Herzog, Ark. Bot. ser. 2, 3: $57.1953=$ Cheilolejeunea subopaca

- Euosmolejeunea serpentina (Mitt.) Steph., Sp. Hepat. 5: 590. $1914=$ Cheilolejeunea serpentina

- Euosmolejeunea trifaria (Reinw., Blume \& Nees) Steph., Hedwigia 29: 78. $1890=$ Cheilolejeune trifaria

Frullania Raddi (Frullaniaceae)

- Frullania apiculata (Reinw., Blume \& Nees) Dumort., Recueil Observ. Jungerm. 13. 1835. AS (Singh and Barbhuiya, 2012).

- Frullania apiculata (Reinw., Blume \& Ness) Dumort. var. obtusata S.Hatt., J. Jap. Bot. 53: 129. $1978=$ Frullania apiculata

- Frullania inuena Steph., Sp. Hepat. 4: 398. $1910=$ Frullania muscicola var. inuena

- Frullania muscicola Steph. var. inuena (Steph.) Kamim., J. Hattori Bot. Lab. 24: 25. 1961. SK (Dey and Singh, 2012).

- Frullania pennsylvanica Steph., Hedwigia 22: 147. $1883=$ Jubula pennsylvanica subsp. pennsylvanica

- Frullania pran-nathii M.Dey \& D.K.Singh, J. Jap. Bot. 83: 281. 2008. WB (Dey and Singh, 2008b, 2012).

- Frullania striata (Lehm. \& Lindenb.) Mont., Ann. Sci. Nat. Ser. 2, 18: 13. 1842 = Ptychanthus striatus

- Frullania tamarisci (L.) Dumort., Recueil observ. Jungerm. 13. 1835 subsp. tamarisci. AR (Dey and Singh, 2012).

- Harpalejeunea indica Steph., Sp. Hepat. 6: 392. 1923 = Lejeunea stevensiana 
- Harpalejeunea ocellata (Herzog) R.M.Schust., Beih. Nova Hedwigia 9: 117. $1963=$ Drepanolejeunea herzogii

Heteroscyphus Schiffn. (Lophocoleaceae)

- Heteroscyphus bescherellei (Steph.) S.Hatt., Bot. Mag. (Tokyo) 58: 39. 1944. SK (Dey and Singh, 2012).

- Heteroscyphus hyalinus (Steph.) Abha Srivast. \& S.C.Srivast., Indian Geocalyaceae 118. 2002. SK (Dey et al., 2009; Dey and Singh, 2012).

- Hygrolejeunea nicobarica Gottsche ex Steph., Sp. Hepat. 5: 564. 1914 = Lejeunea sordida

- Hygrolejeunea obscura (Mitt.) Steph., Sp. Hepat. 5: $565.1914=$ Lejeunea obscura

- Hygrolejeunea sordida (Nees) Steph., Sp. Hepat. 5: 570. $1914=$ Lejeunea sordida

Jubula Dumort. (Jubulaceae)

- Jubula pennsylvanica (Steph.) A.Evans, Rhodora 7: 55. 1905. subsp. Pennsylvanica. SK (Dey et al., 2011; Dey and Singh, 2012).

- Jungermannia apiculata Blume \& Nees, Nova Acta Phy. Med. Acad. Caes. Leop.-Carol. Nat. Cur. 12: 222.825 = Frullania apiculata

- Jungermannia calyptrifolia Hook., Brit. Jungermann. Pl. 43: 1-13.1813 = Colura calyptrifolia

- Jungermannia cucullata Reinw., Blume \& Nees, Nova Acta Phys.-Med. Acad. Caes. Leop.Carol. Nat. Cur. 12: 227. $1825=$ Metalejeunea cucullata

- Jungermannia elliptica Lehm. \& Lindenb. in Lehm., Nov. Stirp. Pug. 5: 13. $1833=$ Leptolejeunea elliptica

- Jungermannia flava Sw., Prodr. 144. $1788=$ Lejeunea flava

- Jungermannia floccosa Lehm. \& Lindenb. in Lehm., Nov. Stirp. Pug. 5: 26. $1833=$ Cololejeunea floccosa

- Jungermannia fulvifolia Hook.f. \& Taylor, London J. Bot. 4: 85.1845 = Radula fulvifolia

- Jungermannia furcata L., Sp. Pl. 1136. $1753=$ Metzgeria furcata var. furcata

- Jungermannia sordida Nees, Enum. Pl. Crypt. Jav. 41. 1830 = Lejeunea sordida

- Jungermannia striata Lehm. \& Lindenb. in Lehm., Nov. Stirp. Pug. 4: 16. $1832=$ Ptychanthus striatus

- Jungermannia tamarisci L., Sp. Pl. 1134. 1753 = Frullania tamarisci subsp. tamarisci

- Jungermannia tenuis Reinw., Blume \& Nees, Nova Acta Acad. Caes. Leop.-Carol. German. Nat. Cur. 12: 226. $1824=$ Drepanolejeunea angustifolia

- Jungermannia thymifolia Nees var. imbricata Nees, Enum. Pl. Crypt. Jav. 42. $1830=$ Cheilolejeunea trapezia

- Jungermannia trapezia Nees, Enum. Pl. Crypt. Jav. 41. $1830=$ Cheilolejeunea trapezia
- Jungermannia trifaria Reinw., Blume \& Nees, Nova Acta Phys.-Med. Acad. Caes. Leop. Carol. Nat. Cur. 12: 226. $1825=$ Cheilolejeune trifaria

- Jungermannia ulicina Taylor, Trans. Proc. Bot. Soc. Edinburgh 1: 115. 1844 = Microlejeunea ulicina

- Jungermannia violacea Ach., F. Weber \& D. Mohr, Beitr. Naturk. 1: 77. $1805=$ Metzgeria violacea

- Jungermannia wallichiana Lehm., Nov. Stirp. Pug. 3: 5.1831 = Microlejeunea wallichiana

Lejeunea Lib. (Lejeuneaceae)

- Lejeunea aequabilis Sande Lac., Ann. Mus. Bot. Lugduno-Batavi 1: 310. 1864 = Cololejeunea aequabilis

- Lejeunea alata Gottsche, in Gottsche, Lindenb. \& Nees, Syn. Hepat. 406. 1845. AR, SK, WB (Dey and Singh, 2008b; Dey and Singh, 2012).

- Lejeunea aloba Sande Lac., Verh. Kon. Ned. Akad. Wetensch. Afd. Natuurk. 5: 72 [Syn. Hepat. Jav.]. 1856. KA (Pande \& Misra, 1942 as Rectolejeunea aloba; Lal, 2003).

- Lejeunea angustifolia Mitt., J. Proc. Linn. Soc., Bot. 5: 116. $1861=$ Drepanolejeunea angustifolia

- Lejeunea anisophylla Mont., Ann. Sci. Nat., Bot., Sér. 2. 19: 263. 1843. AN, AR, SK (Dey et al., 2009; Dey and Singh, 2012; Singh et al., 2016).

- Lejeunea apiculata Sande Lac., Ned. Kruidk. Arch. 3: 421. 1851. SK (Dey et al., 2009 as Stenolejeunea apiculata; Dey and Singh, 2012).

- Lejeunea appendiculata Mitt., J. Proc. Linn. Soc. Bot., 5: 113. $1861=$ Microlejeunea wallichiana

- Lejeunea boninensis Horik., J. Sci. Hiroshima Univ., Ser. B, Div. 2, Bot. 1: 24. $1931=$ Lejeunea anisophylla

- Lejeunea borneensis Steph., Sp. Hepat. 5: 769. 1915 = Lejeunea anisophylla

- Lejeunea cardiocarpa Mont., Hist. Phys. Cuba, Bot., Pl. Cell. 476. $1842=$ Cololejeunea cardiocarpa

- Lejeunea cocoes Mitt., J. Proc. Linn. Soc., Bot. 5: 114. 1861. TN, WB (Pande \& Misra, 1943 as Leptolejeunea himalayensis; Daniels and Daniel, 2013 as Lejeunea himalayensis).

- Lejeunea conica Sande Lac., Ann. Mus. Bot. Lugduno-Batavi 1: 311.1864 = Colura conica

- Lejeunea cucullata (Reinw., Blume \& Nees) Nees, Naturgesch. Eur. Leberm. 3: 293. $1838=$ Metalejeunea cucullata

- Lejeunea curviloba Steph., Sp. Hepat. 5: 774. 1915. SK (Dey and Singh, 2012).

- Lejeunea discreta Lindenb., Gottsche, Lindenb. \& Nees, Syn. Hepat. 361. 1845. SK, TN, WB (Lal, 2003; Dey and Singh, 2012). 
- Lejeunea diversifolia Mitt., J. Proc. Linn. Soc., Bot. 5: 118.1861 = Cololejeunea longifolia

- Lejeunea dozyana Sande Lac., Syn. Hepat. Jav. 63. 1856 = Cololejeunea dozyana

- Lejeunea eifrigii Mizut., J. Hattori Bot. Lab. 33: 244. 1970. SK (Dey et al., 2007; Dey and Singh, 2012).

- Lejeunea epiphylla Mitt., J. Proc. Linn. Soc., Bot. 5: 118. 1861 = Cololejeunea epiphylla

- Lejeunea eulopha Taylor, London J. Bot. 5: 391. 1846 = Lopholejeunea eulopha

- Lejeunea flava (Sw.) Nees, Naturgesch. Eur. Leberm. 3: 277. 1838. AR, AS, MN, SK, WB (Singh et al., 2010a; Dey and Singh, 2012; Singh and Barbhuiya, 2012; Singh and Singh, 2013).

- Lejeunea gilva Gottsche, Abh. Naturwiss. Vereine Bremen 7: 353. 1882 = Acanthocoleus gilvus

- Lejeunea goebelii Gottsche ex K.I. Goebel var. acrotremae Schiffn., Nova Acta Acad. Caes. Leop.-Carol. German. Nat. Cur. 60: 242. 1893 = Cololejeunea jelinekii

- Lejeunea goebelii Gottsche ex K.I.Goebel, Ann. Jard. Bot. Buitenzorg 7: 49. $1888=$ Cololejeunea trichomanis

- Lejeunea gracillima Mitt., J. Proc. Linn. Soc., Bot. 5: 115.1861 = Microlejeunea punctiformis

- Lejeunea haskarliana Lehm. \& Lindenb. in Lehm., Nov. Stirp. Pug. 8: 26. $1844=$ Cololejeunea haskarliana

- Lejeunea himalayensis (Pande \& R.N. Misra) A.E.D.Daniels \& P. Daniel, Bryofl. South. W. Ghats India 205. 2013 = Lejeunea cocoes

- Lejeunea imbricata (Nees) Gottsche, Lindenb. \& Nees, Syn. Hepat. 359. $1845=$ Cheilolejeunea trapezia

- Lejeunea infuscata Mitt., J. Proc. Linn. Soc., Bot. 5: 111. 1861 = Acrolejeunea infuscata

- Lejeunea intertexta Lindenb. in Gottsche et al., Syn. Hepat. 379. $1845=$ Cheilolejeunea intertexta

- Lejeunea kashyapii M.Dey, D.K.Singh \& D. Singh, J. Bryol. 30: 126. 2008. ML, SK (Dey et al., 2008b; Dey and Singh, 2012; Singh et al., 2015).

- Lejeunea kudremukhensis Sushsil K. Singh \& Pócs in Singh et al., Liver. Horn. India Ann. Checkl. 116. 2016. TN (Lal, 2003 as L. tenerrima).

- Lejeunea laeviuscula Mitt., J. Proc. Linn. Soc., Bot. 5: $114.1861=$ Cheilolejeunea laeviuscula

- Lejeunea lehmanniana Gottsche in Gottsche et al., Syn. Hepat. 325. $1845=$ Caudalejeunea lehmanniana

- Lejeunea longifolia Mitt., J. Proc. Linn. Soc., Bot. 5: 117. 1861 = Cololejeunea longifolia
- Lejeunea macounii Spruce ex Underw., Bull. Torrey Bot. Club 17: 259. $1890=$ Cololejeunea macounii

- Lejeunea maculata Mitt., J. Proc. Linn. Soc., Bot. 5: 118. 1861 = Leptolejeunea maculata

- Lejeunea mehrana M.Dey, D.K.Singh \& D. Singh, J. Bryol. 30: 128. 2008. SK (Dey et al., 2008b; Dey and Singh, 2012).

- Lejeunea nigricans Lindenb. in Gottsche et al., Syn. Hepat. 316. $1845=$ Lopholejeunea nigricans

- Lejeunea obscura Mitt., J. Proc. Linn. Soc., Bot. 5: 112. 1861. AN, AR, MN, SK, WB (Singh et al., 2010a; Dey and Singh, 2012; Singh and Barbhuiya, 2012; Singh et al., 2016).

- Lejeunea papilionacea Steph., Hedwigia 31: 17. 1892. SK, WB (Singh et al., 2008; Dey and Singh, 2012).

- Lejeunea parva (S. Hatt.) Mizut., Misc. Bryol. Lichenol. 5: 178. 1971. AS (Singh and Barbhuiya, 2012).

- Lejeunea pentadactyla Mont., Ann. Sci. Nat., Bot., Ser. 3, 10: 113. 1848 = Drepanolejeunea pentadactyla

- Lejeunea planissima Mitt., J. Proc. Linn. Soc., Bot. 5: 117.1861 = Cololejeunea planissima

- Lejeunea planiuscula Mitt., J. Proc. Linn. Soc., Bot. 5: $111.1861=$ Archilejeunea planiuscula

- Lejeunea punctiformis Taylor, London J. Bot. 5: 398. $1846=$ Microlejeunea punctiformis

- Lejeunea sandvicensis (Gottsche) A.Evans, Trans. Connecticut Acad. Arts 8: 253. $1892=$ Acrolejeunea sandvicensis

- Lejeunea schiffneri auct. non Steph. ex Schiffn.: sensu Pande et al., J. Indian Bot. Soc. 36: 338. 1957 = Leptolejeunea elliptica

- Lejeunea serpentina Mitt., J. Proc. Linn. Soc., Bot. 5: $112.1861=$ Cheilolejeunea serpentina

- Lejeunea sordida (Nees) Nees, Naturgesch. Eur. Leberm. 3: 278. 1838. SK (Dey et al., 2009; Dey and Singh, 2012).

- Lejeunea stahliana Steph., Sp. Hepat. 5: 788. 1915 $=$ Lejeunea discreta

- Lejeunea stevensiana (Steph.) Mizut., J. Hattori Bot. Lab. 34: 452. 1971. SK (Singh and Singh, unpubl.).

- Lejeunea subacuta Mitt., J. Proc. Linn. Soc., Bot. 5: 113. 1861. SK, WB (Dey and Singh, 2012).

- Lejeunea subopaca Mitt., J. Proc. Linn. Soc., Bot. 5: $116.1861=$ Cheilolejeunea subopaca

- Lejeunea tenerrima (Steph.) A. Agarwal ex P.K.Verma \& S.C. Srivast., Proc. Natl. Acad. Sci. India, B. 77: 206. 2007, non Lejeunea tenerrima (Nees) Lindenb. 1845, nom. Illeg. = Lejeunea kudremukhensis

- Lejeunea ternatensis Gottsche in Gottsche et al., Syn. Hepat. 346. $1845=$ Drepanolejeunea ternatensis 
- Lejeunea thwaitesiana Mitt., J. Proc. Linn. Soc., Bot. 5: 117. $1861=$ Drepanolejeunea thwaitesiana var. thwaitesiana

- Lejeunea trichomanis Gottsche in Abh. Naturwiss. Vereine Bremen 7: 362.1882 = Cololejeunea trichomanis

- Lejeunea tuberculosa Steph., Sp. Hepat. 5: 790. 1915. AN, AR, AS, MN, SK, WB (Singh et al., 2010a; Singh and Barbhuiya, 2012; Dey and Singh, 2012; Singh et al., 2016).

- Lejeunea ulicina (Taylor) Gottsche in Gottsche et al., Syn. Hepat. 387. 1845 = Microlejeunea ulicina

- Lejeunea vaginata Steph., Sp. Hepat. 5: 791. 1915 $=$ Lejeunea discreta

- Lejeunea venusta Sande Lac., Syn. Hep. Jav. 64. 1856 = Cololejeunea haskarliana

- Lejeunea wallichiana (Lehm.) Lindenb. in Gottsche et al., Syn. Hepat. 361. $1845=$ Microlejeunea wallichiana

Lepidolejeunea R.M.Schust. (Lejeuneaceae)

- Lepidolejeunea bidentula (Steph.) R.M. Schust., Phytologia 45: 425. 1980. AN (Singh et al., 2016).

- Lepidolejeunea nicobarica (Steph.) R.M. Schust., Phytologia 45: 425. 1980 = Lepidolejeunea bidentula

- Leptocolea acrotremae (Schiffn.) Steph., Sp. Hepat. 5: 847. 1916 = Cololejeunea jelinekii

- Leptocolea appressa A.Evans, Bull. Torrey Bot. Club 39: 606. 1912 = Cololejeunea appresa

- Leptocolea ceratilobula P.C. Chen, Feddes Repert. Spec. Nov. Regni Veg. 58: 49. $1955=$ Cololejeunea ceratilobula

- Leptocolea distalopapillata E.W.Jones, Trans. Brit. Bryol. Soc. 3: 202. $1957=$ Cololejeunea distalopapillata

- Leptocolea furcilobulata Berrie \& E.W.Jones, Trans. Brit. Bryol. Soc. 2: 417. $1954=$ Cololejeunea furcilobulata

- Leptocolea himalayensis Pande \& R.N.Misra, Proc. Nat. Acad. India 13 (1): $27.1943=$ Cololejeunea latilobula

- Leptocolea hispidissima Steph., Sp. Hepat. 6: 423. 1923 = Cololejeunea haskarliana

- Leptocolea jelinekii (Steph.) Steph., Sp. Hepat. 5: 851. 1916 = Cololejeunea jelinekii

- Leptocolea lanciloba (Steph.) A. Evans, Bull. Torrey Bot. Club 38: 268. $1911=$ Cololejeunea lanciloba

- Leptocolea latilobula Herzog in Hand.-Mazz., Symb. Sin. 5: 54. $1930=$ Cololejeunea latilobula

- Leptocolea ocellata Horik., J. Sci. Hiroshima Univ., Ser. B, Div. 2, Bot. 1: 86. $1932=$ Cololejeunea ocellata
- Leptocolea pseudofloccosa Horik., J. Sci. Hiroshima Univ., Ser. B, Div. 2, Bot. 1: 87. 1932 = Cololejeunea pseudofloccosa

Leptolejeunea (Spruce) Schiffn. (Lejeuneaceae)

- Leptolejeunea apiculata (Horik.) S.Hatt., J. Hattori Bot. Lab. 5: 46. 1951. AR, SK (Singh et al., 2010c; Dey and Singh, 2012).

- Leptolejeunea balansae Steph., Hedwigia 35: 105. 1896. AN, AR, AS, MN, SK, TN, WB (Awasthi, 1986; Lal, 2003; Daniels and Daniel, 2004; Singh et al., 2010a, b; Singh and Barbhuiya, 2012; Dey and Singh, 2012; Daniels and Daniel, 2013).

- Leptolejeunea dapitana Steph., Bull. Herb. Boissier 5: 79. $1897=$ Leptolejeunea elliptica

- Leptolejeunea elliptica (Lehm. \& Lindenb.) Schiffn. in Engler \& Prantl, Nat. Pflanzenfam. 1(3): 126. 1893. AN, AR, AS, MN, ML, SK, TN, WB (Lal, 2003 as $L$. subacuta; Singh and Barbhuiya, 2012, Dey and Singh, 2012; Daniels and Daniel, 2013).

- Leptolejeunea epiphylla (Mitt.) Steph., Sp. Hepat. 5: 380. 1914. AN (Lal, 2003; Singh et al., 2006a, 2010b, 2016).

- Leptolejeunea erecta Steph., Bull. Soc. Roy. Bot. Belgique 38: 44. 1899 = Drepanolejeunea erecta

- Leptolejeunea fleischeri Steph., Sp. Hepat. 5: 382. 1913 = Drepanolejeunea fleischeri

- Leptolejeunea foliicola auct. (Horik.) R.M. Schust., Beih. Nova Hedwigia. 9: 116. 1963, non Leptolejeunea foliicola Steph. = Drepanolejeunea foliicola

- Leptolejeunea foliicola Steph., Hedwigia 35: 106. 1896. AR, KL, ML (Udar and Awasthi, 1982; Singh 1996; Lal, 2003; Dey and Singh, 2012; Manju et al., 2012).

- Leptolejeunea himalayensis Pande \& R.N.Misra, J. Indian Bot. Soc. 22: 168. 1943 = Lejeunea cocoes

- Leptolejeunea latifolia Herzog, Mem. Soc. Fauna Flora Fenn. 26: 58. 1950. SK, WB (Dey and Singh, 2012).

- Leptolejeunea maculata (Mitt.) Schiffn., Consp. Hepat. Archip. Ind. 275. 1898. AN, KA, WB, TN (Lal, 2003 as Leptolejeunea schiffneri; Singh et al., 2006a; Singh et al., 2010b; Daniels and Daniel, 2013; Singh et al., 2016).

- Leptolejeunea mirikana M.Dey \& D.K.Singh, Taiwania 55: 355. 2010. WB (Dey and Singh, 2010b, 2012).

- Leptolejeunea schiffneri auct. non Steph.: sensu Pande et al., J. Indian Bot. Soc. 36: 338. 1957 = Leptolejeunea elliptica

- Leptolejeunea schiffneri (Steph. ex Schiffn.) Steph., Hedwigia 35: 107. $1896=$ Leptolejeunea maculata 
- Leptolejeunea sikkimensis Udar \& U.S.Awasthi, Misc. Bryol. Lichenol. 8: 6. 1979. TN (Daniels and Daniel, 2013).

- Leptolejeunea subacuta Steph. ex A.Evans, Proc. Wash. Acad. Arts Sci. 8: 149. $1906=$ Leptolejeunea elliptica

- Leptolejeunea subdentata Schiffn. ex Herzog in Flora 135: 403. 1942. AR, SK, WB (Singh et al., 2006b; Dey and Singh, 2012).

- Leptolejeunea udarii M.Dey \& D.K.Singh, Taiwania 55: 359. 2010. SK (Dey and Singh, 2010b, 2012).

- Lophocolea hyalina Steph., Bull. Acad. Soc. Roy. Sci. Belgique 38: 254. 1899 = Heteroscyphus hyalinus

Lopholejeunea (Spruce) Schiffn. (Lejeuneaceae)

- Lopholejeunea eulopha (Taylor) Schiffn. in Engler \& Prantl, Nat. Pflanzenfam. 1 (3): 129. 1895. AS (Singh and Barbhuiya, 2012).

- Lopholejeunea nicobarica Steph., Hedwigia 35: 111. 1896 = Lopholejeunea eulopha

- Lopholejeunea nigricans (Lindenb.) Schiffn., Consp. Hepat. Arch. Indici 293. 1898. AS (Singh and Barbhuiya, 2012).

- Lopholejeunea sikkimensis Steph., Sp. Hepat. 5: 87. 1912. var. sikkimensis. AN, AS, SK (Singh and Barbhuiya, 2012; Singh et al., 2014 as Lopholejeunea nigricans; Singh et al., 2016).

- Lopholejeunea udarii M.Dey \& D.K.Singh, Nelumbo 53: 197. 2011. AR (Dey and Singh, 2011b, 2012).

- Marchesinia sikkimensis Steph., Sp. Hepat. 5: 155. 1912 = Acrolejeunea infuscata

- Mastigolejeunea sandvicensis (Gottsche) Steph., Bull. Herb. Boissier 5: 842. $1897=$ Acrolejeunea sandvicensis

Metalejeunea Grolle (Lejeuneaceae)

- Metalejeunea cucullata (Reinw., Blume \& Nees) Grolle, Bryophyt. Biblioth. 48: 100. 1995. TN (Verma et al., 2013).

Metzgeria Raddi (Metzgeriaceae)

- Metzgeria conjugata Lindb. supsp. japonica (S. Hatt.) Kuwah., J. Hattori Bot. Lab. 20: 135. $1958=$ Metzgeria lindbergii

- Metzgeria conjugata Lindb. var. japonica S. Hatt., J. Hattori. Bot. Lab. 15: 80.1955 = Metzgeria lindbergii

- Metzgeria conjugata Lindb., Acta Soc. Sci. Fenn. 10: 495. 1875. AR, SK (Singh et al., 2007; Singh and Singh, 2008; Dey and Singh, 2012).

- Metzgeria consanguinea Schiffn., Nova Acta Acad. Caes. Leop.-Carol. German. Nat. Cur. 60: 271. 1893. AR, SK (Singh et al., 2007; Dey and Singh, 2012).

- Metzgeria crispula Herzog, Ann. Bryol. 12: 72. $1939=$ Metzgeria furcata var. furcata
- Metzgeria darjeelingensis Kuwah., J. Hattori Bot. Lab. 39: 365. 1975, p.p.= Metzgeria consanguinea

- Metzgeria decipiens (C.Massal.) Schiffn., Forschungsr. Gazelle 4: 43. 1890 = Metzgeria furcata var. furcata

- Metzgeria fruticulosa (Dicks.) A. Evans, Ann. Bot. 24: 296. 1910 = Metzgeria violacea

- Metzgeria furcata (L.) Corda var. decipiens C.Massal., Nuovo Giorn. Bot. Ital. 17:256. 1885 = Metzgeria furcata var. furcata

- Metzgeria furcata (L.) Dumort. Naturalientausch 12: 654. 1829 var. furcata. AR, SK, WB (Singh et al., 2007; Dey and Singh, 2012).

- Metzgeria furcata (L.) Dumort. var. ulvula Nees, Naturgesch. Eur. Leberm. 3: 489. 1838. AR, SK, WB (Singh et al., 2007; Dey and Singh, 2012).

- Metzgeria furcata f. ulvula Nees, Naturgessch. Eur. Leberm. 3: 489. $1838=$ Metzgeria furcata var. ulvula

- Metzgeria lindbergii Schiffn., Denkschr. Kaiserl. Akad. Wiss., Math.-Naturwiss. Kl. 67: 182.1898. AR, SK, WB (Singh et al., 2007; Dey and Singh, 2012).

- Metzgeria longitexta Steph., Bull. Herb. Boissier 7: 940. 1899 \& Sp. Hepat. 1: $288.1899=$ Metzgeria consanguinea

- Metzgeria lutescens Steph., Sp. Hepat. 6: 54. 1917 = Metzgeria furcata var. furcata

- Metzgeria nilgiriensis Udar \& S.C.Srivast., New Botanist 2: 24.1975. MN, TN (Lal, 2003).

- Metzgeria sikkimensis S.C.Srivast. \& K.K.Rawat, Geophytology 31: 71. 2003. SK (Singh et al., 2007; Dey and Singh, 2012).

- Metzgeria temperata Kuwah., J. Hattori Bot. Lab. 40: 219. 1976. SK (Singh et al., 2007; Dey and Singh, 2012).

- Metzgeria violacea (Ach.) Dumort., Jungerm. 26. 1835. SK (Singh et al., 2007; Dey and Singh, 2012).

Microlejeunea Steph. (Lejeuneaceae)

- Microlejeunea gracillima (Mitt.) Carr. \& Pears, Proc. Linn. Soc. N.S. Wales Ser. 2, 2: 1040. $1888=$ Microlejeunea punctiformis

- Microlejeunea longirostris Steph., Sp. Hepat. 6: 422. 1923 = Microlejeunea wallichiana

- Microlejeunea minutistipula Steph., Hedwigia 29: 89. 1890. AN (Lal, 2003).

- Microlejeunea punctiformis (Taylor) Steph., Hedwigia 29: 90. 1890. AR, SK, TN, WB (Dey and Singh, 2012; Verma et al., 2013; Singh and Singh, 2013).

- Microlejeunea rotundistipula f. parva S. Hatt., Bull. Tokyo Sci. Mus. 11: 123. $1944=$ Lejeunea parva 
- Microlejeunea ulicina (Taylor) A. Evans, Mem. Torrey Bot. Club 8: 162. 1902. nom. inval. = Microlejeunea ulicina

- Microlejeunea ulicina (Taylor) Steph., Hedwigia 29: 88. 1890. AS, ML, WB, TN (Lal, 2003; Singh and Barbhuiya, 2012).

- Microlejeunea wallichiana (Lehm.) R.L.Zhu \& Y.M.Wei, Cryptog. Bryol. 34: 308. 2013. SK, WB (Dey and Singh, 2012; Singh and Singh, 2013, both as Lejeunea wallichiana).

- Phragmicoma reniloba Gottsche in Gottsche et al., Syn. Hepat. 301. $1845=$ Caudalejeunea reniloba

- Phragmicoma sandvicensis Gottsche, Ann. Sci. Nat., Bot., Ser. 4, 8: 344.1857 = Acrolejeunea sandvicensis

- Physocolea acinacifolia Steph., Sp. Hepat. 5: 887. 1916 = Cololejeunea longifolia

- Physocolea angustifolia (Mitt.) Steph., Sp. Hepat. 5: $887 . \quad 1916=$ Drepanolejeunea angustifolia

- Physocolea bolombensis Steph., Sp. Hepat. 5: 868. 1916 = Cololejeunea lanciloba

- Physocolea denticulata Horik., J. Sci. Hiroshima Univ., Ser. B, Div. 2, Bot. 2: 287. 1934 = Cololejeunea denticulata

- Physocolea diversifolia (Mitt.) Steph., Sp. Hepat. 5: 892. 1916 = Cololejeunea longifolia

- Physocolea dozyana (Sande Lac.) Steph., Sp. Hepat. 5: 891. 1916 = Cololejeunea dozyana

- Physocolea gottschei Steph., Sp. Hepat. 5: 894. 1916 = Cololejeunea gottschei

- Physocolea hispidissima (Steph.) Herzog, Ann. Bryol. 4: $94.1931=$ Cololejeunea haskarliana

- Physocolea longifolia (Mitt.) Steph., Sp. Hepat. 5: 898. 1916 = Cololejeunea longifolia

- Physocolea madothecoides Steph., Sp. Hepat. 5: 898. 1916 = Cololejeunea madothecoides

- Physocolea raduliloba (Steph.) Steph., Sp. Hepat. 5: 903.1916 = Cololejeunea raduliloba

- Physocolea serrulata Steph., Sp. Hepat. 5: 906. 1916 = Cololejeunea serrulata

- Physocolea spinosa Horik., J. Sci. Hiroshima Univ., Ser. B, Div. 2, Bot. 1: 70. $1931=$ Cololejeunea spinosa

- Physocolea trianguliloba Steph., Sp. Hepat. 5: 907. 1916 = Cololejeunea peraffinis

- Physocolea trichomanis (Gottsche) Steph., Sp. Hepat. 5: 912. $1916=$ Cololejeunea trichomanis

Plagiochila (Dumort.) Dumort. (Plagiochilaceae)

- Plagiochila acanthophylla Gottsche, Bot. Zeitung (Berlin) 16, Beil. 37: 38. 1858 = Plagiochila sciophila

- Plagiochila brevifolia Steph., Bull. Herb. Boissier, Ser. 2, 3: 876. 1903 = Plagiochila nepalensis
- Plagiochila chiloscyphoidea Steph., Bull. Herb. Boissier, Ser. 2, 3: 121. 1903 = Plagiochila sciophila

- Plagiochila cornuta Steph., Bull. Herb. Boissier, Ser. 2, 3: 874. 1903 \& Sp. Hepat. 2: 359. $1903=$ Plagiochila nepalensis

- Plagiochila cuspidata Steph., Sp. Hepat. 6: 144. 1918 = Plagiochila parvifolia

- Plagiochila decolyana Schiffn. ex Steph., Sp. Hepat. 6: 144. 1918 = Plagiochila nepalensis

- Plagiochila denticulata Mitt., J. Proc. Linn. Soc., Bot. 5: 95. 1861. SK (Dey and Singh, 2012).

- Plagiochila dichotomoramosa Inoue, J. Hattori Bot. Lab. 30: 125. 1967 = Plagiochila fordiana

- Plagiochila flavovirens Steph., Sp. Hepat. 6: 156. 1918 = Plagiochila sciophila

- Plagiochila fordiana Steph., Bull. Herb. Boissier (ser. 2) 3: 104. 1902. AS (Singh and Barbhuiya, 2012).

- Plagiochila furcifolia Mitt., Trans. Linn. Soc. London, Bot. ser 2, 3: 194. 1891. AS (Singh and Barbhuiya, 2012).

- Plagiochila gammiana Steph., Bull. Herb. Boissier, Ser. 2, 3: 963. 1903 \& Sp. Hepat. 2: 378. 1903 = Plagiochila nepalensis

- Plagiochila gollanii Steph., Bull. Herb. Boissier, Ser. 2, 3: 883. 1903 \& Sp. Hepat. 2: 368. $1903=$ Plagiochila nepalensis

- Plagiochila gollanii var. triquetra Herzog, Ann. Bryol. 12: 76. 1939 = Plagiochila nepalensis

- Plagiochila grata Steph., Sp. Hepat. 6: 160. $1918=$ Plagiochila nepalensis

- Plagiochila horridula Steph., Sp. Hepat. 6: 168. 1918 = Plagiochila denticulata

- Plagiochila luethiana Steph., Sp. Hepat. 6: 180. 1921 = Plagiochila nepalensis

- Plagiochila neorupicola Inoue, Bryologist 68: 218. 1965 = Plagiochila parvifolia

- Plagiochila nepalensis Lindenb., Sp. Hepat. (Plagiochila fasc. 2 - 4): 93. 1840. AR (Dey and Singh, 2012).

- Plagiochila orientalis Taylor, London J. Bot. 5: 261. 1846 = Plagiochila sciophila

- Plagiochila parvifolia Lindenb., Sp. Hepat. (Plagiochila fasc. 1): 28.1839. AS (Singh and Barbhuiya, 2012).

- Plagiochila phalangea Taylor, London J. Bot. 5: 264. 1846 = Plagiochila parvifolia

- Plagiochila pseudorientalis Inoue, J. Hattori Bot. 30: 126.1967 = Plagiochila nepalensis

- Plagiochila remotistipula Steph., Sp. Hepat. 6: 201. 1921 = Plagiochila nepalensis

- Plagiochila sciophila Nees ex Lindenb., Sp. Hepat. (Plagiochila fasc. 2 - 4): 100. 1840. AR, SK (Dey and Singh, 2012). 
- Plagiochila semiaperta Schiffn. ex Steph., Sp. Hepat. 6: 210. 1921 = Plagiochila nepalensis

- Plagiochila subsymmetrica Steph., Sp. Hepat. 6: 212. 1921 = Plagiochila parvifolia

- Plagiochila trochantha Schiffn. ex Steph., Sp. Hepat. 6: 226. 1921 = Plagiochila sciophila

- Plagiochila ventricosa Steph., Bull. Herb. Boissier, Ser. 2, 3: 964.1903 \& Sp. Hepat. 2: 379. 1903 = Plagiochila parvifolia

- Plagiochila vygensis Steph., Sp. Hepat. 6: 237. 1921 = Plagiochila sciophila

- Plagiochila yokogurensis Taylor, Bull. Herb. Boissier 5: $104.1897=$ Plagiochila parvifolia

Ptychanthus Nees (Lejeuneaceae)

- Ptychanthus argutus Steph., Sp. Hepat. 4: 745. $1912=$ Ptychanthus striatus

- Ptychanthus birmensis (Steph.) Steph., Sp. Hepat. 4: 745. 1912 = Ptychanthus striatus

- Ptychanthus chinensis Steph., Sp. Hepat. 4: 744. 1912 = Ptychanthus striatus

- Ptychanthus effusus Steph., Sp. Hepat. 4: 746. 1912 = Ptychanthus striatus

- Ptychanthus nietneri (Steph.) Schiffn., Oesterr. Bot. Z. 49: 205. 1899 = Ptychanthus striatus

- Ptychanthus perrottetii (Steph.) Steph., Sp. Hepat. 4: 750.1912 = Ptychanthus striatus

- Ptychanthus pyriformis (Steph.) Steph., Sp. Hepat. 4: 751.1912 = Ptychanthus striatus

- Ptychanthus striatus (Lehm. \& Lindenb.) Nees, Naturgesch. eur. Leberm. 3: 212. 1838. AR, SK, WB (Dey and Singh, 2012).

- Ptychanthus striatus var. perrottettii (Steph.) Verd., Ann. Bryol. Suppl. 4: 121. $1934=$ Ptychanthus striatus

- Ptychanthus striatus var. retusus (Reinw., Blume \& Nees) Verd., Ann. Bryol. Suppl. 4: 121.1934 $=$ Ptychanthus striatus

- Ptychanthus wightii Gottsche in Gottsche et al., Syn. Hepat. 291. $1845=$ Ptychanthus striatus

- Ptychocoleus cucullatus (Reinw., Blume \& Nees) Steph., Sp. Hepat. 5: 41. $1912=$ Metalejeunea cucullata

- Ptychocoleus planiusculus (Mitt.) Verd., Ann. Bryol. Suppl. 4: 126. $1934=$ Cheilolejeunea laeviuscula

- Ptychocoleus saccatus (Mitt.) Steph., Sp. Hepat. 5: 53. 1912 = Acrolejeunea infuscata

- Ptycholejeunea birmensis Steph., Hedwigia 35: 120. $1896=$ Ptychanthus striatus

- Ptycholejeunea perrotteti Steph., Hedwigia 35: 121. 1896 = Ptychanthus striatus

- Ptycholejeunea pyriformis Steph., Hedwigia 35: 122. 1896 = Ptychanthus striatus
- Pycnolejeunea bidentula Steph. in Jack \& Steph., Bot. Centralbl. 60: 107. $1894=$ Lepidolejeunea bidentula

- Pycnolejeunea imbricata (Nees) Schiffn., Hepat. (Engl.-Prantl) 124. 1895 = Cheilolejeunea trapezia

- Pycnolejeunea longiloba G. Hoffm., Ann. Bryol. 8: 114. 1935 = Cheilolejeunea trapezia

- Pycnolejeunea nicobarica Steph., Hedwigia 35: 126. 1896 = Lepidolejeunea bidentula

- Pycnolejeunea planifolia (Steph.) G.Hoffm., Ann. Bryol. 8: $122.1935=$ Cheilolejeunea trapezia

Radula Dumort. (Radulaceae)

- Radula acuminata Steph., Sp. Hepat. 4: 230. 1910. AN, AR, AS, ML, MN, SK (Udar and Kumar, 1984; Singh, 1996; Lal, 2003; Singh et al., 2010a; Dey and Singh, 2012; Singh et al., 2016).

- Radula assamica Steph., Hedwigia 23: 151. 1884. AR, AS (Udar and Kumar, 1984; Singh, 1996; Lal, 2003; Dey and Singh, 2012).

- Radula auriculata Steph., Bull. Herb. Boissier 5: 105. 1897. AN, SK (Dey and Singh, 2012; Singh et al., 2016).

- Radula foreauana Beauverd ex Steph., Sp. Hepat. 6: 514.1924 = Radula fulvifolia

- Radula fulvifolia (Hook.F. \& Taylor) Gottsche, Lindenb. \& Nees, Syn. Hepat. 261. 1845. SK (Dey and Singh, 2012).

- Radula javanica Gottsche in Gottsche, Lindenb. \& Nees, Syn. Hepat. 257. 1845. AN, AS, ML, TN (Lal, 2003; Singh et al., 2016).

- Radula meyeri Steph., Hedwigia 27: 62. 1888 = Radula fulvifolia

- Radula multiflora Gottsche ex Schiffn., Forschungsr. Gazelle 4: 20. $1890=$ Radula javanica

- Radula nymanii Steph., Sp. Hepat. 4: 229. 1910. AN (Singh et al., 2016).

- Radula obscura Mitt., J. Proc. Linn. Soc., Bot. 5: 107. 1861. NL, SK (Dey and Singh, 2012; Eshuo et al., 2013).

- Radula protensa Lindenb., Bot. Zeitung (Berlin) 6: 462. 1848. AN, KA (Udar and Kumar, 1984; Lal, 2003; Singh et al., 2016).

- Radula sandei Steph., Hedwigia 9: 130. $1884=$ Radula javanica

- Radula tjibodensis K.I.Goebel, Ann. Jard. Bot. Buitenzorg 7: 53. 1888. AN, AR, ML, MN, SK, WB (Udar and Kumar, 1984; Singh et al., 2010a; Dey and Singh, 2012; Singh et al., 2016).

- Radula wallichiana Lehm., Nov. Stirp. Pug. 10: 9. 1857 = Radula javanica

- Rectolejeunea aloba (Sande Lac.) Steph., Sp. Hepat. 5: 696. 1914 = Lejeunea aloba 
- Rhaphidolejeunea foliicola (Horik.) P.C.Chen, Feddes Repert. Spec. Nov. Regni Veg. 58: 45. 1955 = Drepanolejeunea foliicola

- Rhaphidolejeunea siamensis Bischl., Rev. Bryol. Lichénol. 36: 86. 1969 = Drepanolejeunea siamensis

- Rhaphidolejeunea tibetana P.C.Wu \& J.S.Lou, Acta Phytotax. Sin. 16: 102. $1978=$ Drepanolejeunea tibetana

- Rhaphidolejeunea yunnanensis P.C.Chen, Feddes Repert. Spec. Nov. Regni Veg. 58: 44. $1955=$ Drepanolejeunea yunnanensis

Riccardia Gray (Aneuraceae)

- Riccardia levieri Schiffn., Oesterr. Bot. Z. 49: 130. 1899. KA (Srivastava, 1960).

- Spruceanthus wiggintonii A. E. D. Daniels, K. C. Kariappa \& P. Daniel, Cryptog. Bryol. 31: 121. 2010. (syn. fide Wang et al., 2014) = Ptychanthus striatus

- Stenolejeunea apiculata (Sande Lac.) R.M.Schust., Beih. Nova Hedwigia 9: 144. 1963 = Lejeunea apiculata

- Strepsilejeunea monophthalma Herzog in Hand.Mazz., Symb. Sin. 5: 47. $1930=$ Drepanolejeunea erecta

- Strepsilejeunea ocellata Herzog, Memoranda Soc. Fauna Fl. Fenn. 26: 57. $1950=$ Drepanolejeunea herzogii

- Strepsilejeunea papillata (Herzog) R.M.Schust. \& Kachroo in Kachroo \& R.M. Schust., J. Linn. Soc., Bot. 56: 510. $1961=$ Cheilolejeunea subopaca

- Strepsilejeunea papillata Herzog, Ann. Bryol. 12: $85.1939=$ Cheilolejeunea subopaca

- Strepsilejeunea planifolia Steph., Sp. Hepat. 6: 396. 1923 = Cheilolejeunea trapezia

- Taeniolejeunea peraffinis auct. non (Schiffn.) Zwickel: sensu Pande et al., J. Indian Bot. Soc. 36: 336. 1957 = Cololejeunea appresa

- Taeniolejeunea pseudofloccosa (Horik.) S.Hatt., J. Jap. Bot. 17: 465. $1941=$ Cololejeunea pseudofloccosa

- Taxilejeunea laxiretis (Steph.) Eifrig, Ann. Bryol. 9: 102.1936 = Lejeunea sordida

- Taxilejeunea obscura (Mitt.) Eifrig, Ann. Bryol. 9: 93. 1936 = Lejeunea obscura

- Taxilejeunea sordida (Nees) Eifrig, Ann. Bryol. 9: 101. 1936 = Lejeunea sordida

- Taxilejeunea stevensiana Steph., Hedwigia 35: 136. 1896 = Lejeunea stevensiana

- Taxilejeunea tenerrima Steph., Sp. Hepat. 6: 406. 1923 = Lejeunea kudremukhensis

- Thysananthus renilobus (Gottsche) Schiffn., Consp. Hepat. Arch. Ind. 306. $1898=$ Caudalejeunea reniloba

- Trocholejeunea infuscata (Mitt.) Verd., Ann. Bryol. Suppl. 4: 190. 1934 = Acrolejeunea infuscata
- Trocholejeunea sandvicensis (Gottsche) Mizut., Misc. Bryol. Lichenol. 2: 169. 1962 “1961" = Acrolejeunea sandvicensis

Tuyamaella S.Hatt.

- Tuyamaella serratistipa S.Hatt., Bot. Mag. Tokyo 64: 118. 1951. AR (Singh and Dey, 2010; Dey and Singh, 2012).

\section{Distribution of epiphyllous liverworts}

The epiphyllous liverworts show maximum diversity in eastern Himalaya, including the north-east, with 133 taxa. Of these, 91 taxa are restricted to this region in Indian liverwort flora. This is followed by the Western Ghats with 47 taxa of which 20 are restricted to this territory alone in India, whereas Andman \& Nicobar Islands have 34 taxa of which 13 are restricted to this region. While, seven taxa have been found to grow in all the three brogeographical regions, 18 taxa are common between Eastern Himalaya and Western Ghats, 11 between Eastern Himalaya and Andman \& Nicobar Islands, and only Cololejeunea gottschei and Drepanolejeunea pentadactyla are common between Western Ghats and the Andman \& Nicobar Islands (Fig. 2).

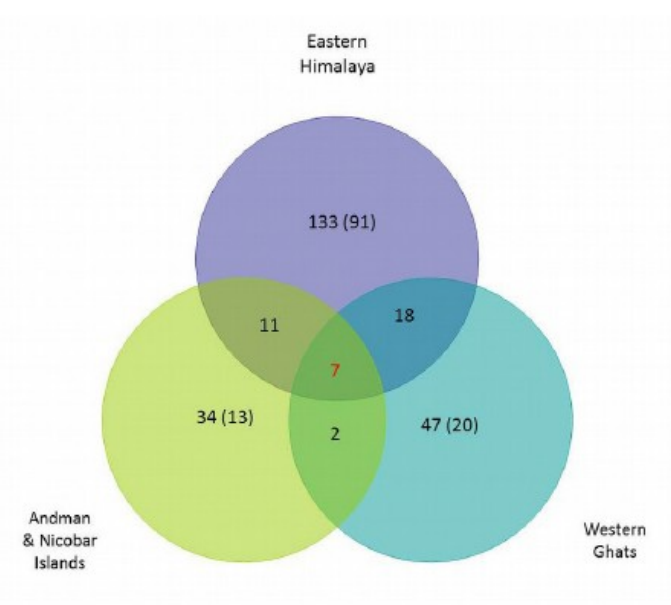

Fig. 2. Bryogeographical region-wise distribution of epiphyllous liverworts in India

Sikkim with 80 taxa shows the maximum diversity of epiphyllous liverworts, followed by Arunachal Pradesh with 57 taxa, West Bengal with 45 taxa, Andman \& Nicobar Islands with 34 taxa, Assam with 30 taxa, Tamil Nadu with 27 taxa, Karnataka with 23 taxa, Manipur with 17 taxa, Meghalaya with 12 taxa, Kerala with seven taxa, Mizoram with four taxa and Nagaland with just a single taxon (Fig. 3).

\section{Endemism}

Twenty species of epiphyllous liverworts are endemic to India. Of these, 11 species are confined to the eastern Himalayan 
bryogeographical region, Cololejeunea karnatakensis, Lejeunea kudremukhensis and Leptolejeunea sikkimensis are restricted to Western Ghats, while only Cololejeunea andmanansis is so far known as endemic only to Andman \& Nicobar Islands. Cololejeunea epiphylla, C. foliicola, C. kashyapii, C. udarii and Metzgeria nilgiriensis are common between eastern Himalaya and the Western Ghats (Table 2).

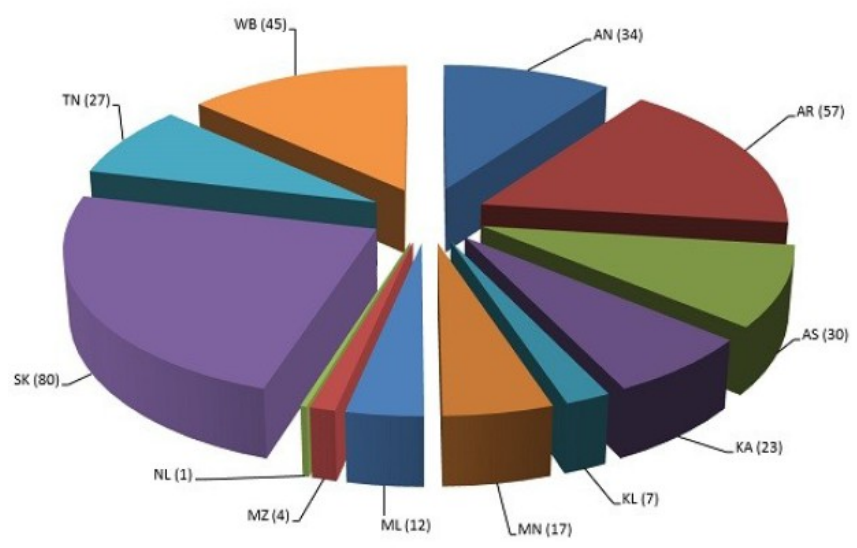

Fig. 3. State-wise distribution of epiphyllous liverworts in India

\section{Acknowledegements}

One of the authors (DS) thanks the Director, Botanical Survey of India for facilities and encouragement.

\section{References}

Asthana, G. and A. Shukla. 2009. Two epiphyllous species of Drepanolejeunea (Spruce) Schiffn. new to the Indian bryoflora. J. Bryol. 31: 139-142.

Asthana, G. and A. Shukla. 2010. Colura leratii (Steph.) Steph. new to India. Tropical Bryol. 32: 100-102.

Asthana, G. and S.C. Srivastava. 2003. Indian Cololejeunea. A taxonomic study. Bryophyt. Biblioth. 60: 1-155.

Awasthi, U.S. 1986. The genus Leptolejeunea (Spruce) Steph. in India. J. Indian Bot. Soc. 65: 117-123.

Bridson, G.D.R., S.T. Townsend, E.A. Polen and E.R. Smith. 2004a. BPH - 2. Periodicals with botanical content. 1 . Hunt Institute for Botanical Documentation, Pittsburgh. pp. 819.

Bridson, G.D.R., S.T. Townsend, E.A. Polen and E.R. Smith 2004b. BPH - 2. Periodicals with botanical content. 2. Hunt Institute for Botanical Documentation, Pittsburgh. pp. 1470.

Brummitt, R.K. and C.E. Powell. 1992. Authors of plant names. Royal Botanic Gardens, Kew. pp. 732.

Crandall-Stotler, B. R.E. Stotler and D.G. Long. 2009. Phylogeny and classification of the Marchantiophyta. Edinburgh J. Bot. 66: 155-198. doi: 10.1017/S0960428609005393

Table. 2. List of the endemic epiphyllous liverworts in India

\begin{tabular}{|c|c|c|c|c|c|}
\hline \multirow{2}{*}{ Sl. no. } & \multirow{2}{*}{ Name of the species } & \multirow{2}{*}{ Family } & \multicolumn{3}{|c|}{ Distribution } \\
\hline & & & EH & WG & AN \\
\hline 1. & Cololejeunea andamanansis M.Dey \& D.K.Singh & Lejeuneaceae & - & - & + \\
\hline 2. & Cololejeunea epiphylla G. Asthana \& A. Shukla & Lejeuneaceae & + & + & - \\
\hline 3. & Cololejeunea foliicola S.C.Srivast. \& G. Srivast. & Lejeuneaceae & + & + & - \\
\hline 4. & Cololejeunea karnatakensis G.Asthana \& S.C.Srivast. & Lejeuneaceae & - & + & - \\
\hline 5. & Cololejeunea kashyapii Udar \& G. Srivast. & Lejeuneaceae & + & + & - \\
\hline 6. & Cololejeunea mizutaniana Udar \& G. Srivast. & Lejeuneaceae & + & - & - \\
\hline 7. & Cololejeunea nilgiriensis G. Asthana \& S.C. Srivast. & Lejeuneaceae & + & - & - \\
\hline 8. & Cololejeunea siangensis G.Asthana \& S.C.Srivast. & Lejeuneaceae & + & - & - \\
\hline 9. & Cololejeunea tixieriana M.Dey, D. Singh \& D.K.Singh & Lejeuneaceae & + & - & - \\
\hline 10. & Cololejeunea udarii G.Asthana \& S.C.Srivast. & Lejeuneaceae & + & + & - \\
\hline 11. & Drepanolejeunea devendrae Sushil K. Singh \& M. Dey & Lejeuneaceae & + & - & - \\
\hline 12. & Frullania pran-nathii M.Dey \& D.K.Singh & Frullaniaceae & + & - & - \\
\hline 13. & Lejeunea kashyapii M.Dey, D.K.Singh \& D. Singh & Lejeuneaceae & + & - & - \\
\hline 14. & Lejeunea kudremukhensis Sushsil K. Singh \& Pócs & Lejeuneaceae & - & + & - \\
\hline 15. & Lejeunea mehrana M.Dey, D.K.Singh \& D. Singh & Lejeuneaceae & + & - & - \\
\hline 16. & Leptolejeunea mirikana M.Dey \& D.K.Singh & Lejeuneaceae & + & - & - \\
\hline 17. & Leptolejeunea sikkimensis Udar \& U.S.Awasthi & Lejeuneaceae & - & + & - \\
\hline 18. & Lopholejeunea udarii M.Dey \& D.K.Singh & Lejeuneaceae & + & - & - \\
\hline 19. & Metzgeria nilgiriensis Udar \& S.C.Srivast. & Metzgeriaceae & + & + & - \\
\hline 20. & Metzgeria sikkimensis S.C.Srivast. \& K.K.Rawat & Metzgeriaceae & + & - & - \\
\hline
\end{tabular}


Daniels, A.E.D. and P. Daniel. 2004. Leptolejeunea balansae Steph. (Hepaticae: Jungermanniales) - a new record of bryoflora from the Indian mainland. J. Bombay Nat. Hist. Soc. 101: 333-334.

Daniels, A.E.D. and P. Daniel. 2009. Cololejeunea distalopapillata and $C$. vidaliana (Lejeuneaceae) new to the liverwort flora of India. Acta. Bot. Hung. 51: 61-66. doi: 10.1556/ABot.51.2009.1-2.8

Daniels, A.E.D. and P. Daniel. 2013. The bryoflora of the Southeastern Western Ghats, India. Bishen Singh Mahendra Pal Singh, Dehra Dun. pp. 352.

Das, S. and D.K. Singh. 2009. Three new records of liverworts for Himalayan region from Mehao Wildlife Sanctuary, Arunachal Pradesh. Nelumbo 51: 191-198.

Dey, M. and D.K. Singh. 2007. Cololejeunea yipii R.L.Zhu (Hepaticae: Lejeuneaceae) - new to Indian bryoflora from West Siang, Arunachal Pradesh. Phytotaxonomy 7: 35-37.

Dey, M. and D.K. Singh. 2008a. Cololejeunea longiana Grolle \& Mizut. - an addition to Indian bryoflora from Darjeeling, West Bengal. Bull. Bot. Surv. India 50: 209-211.

Dey, M. and D.K. Singh. 2008b. Frullania pran-nathii - a new epiphyllous liverwort from Darjeeling, India. J. Jap. Bot. 83: 280-283.

Dey, M. and D.K. Singh. 2008c. Lejeunea alata Gottsche (Lejeuneaceae) - a new record for Indian bryoflora from Arunachal Pradesh. Geophytology 37: 43-45.

Dey, M. and D.K. Singh. 2009. Caudalejeunea lehmanniana (Gottsche) A.Evans (Hepaticae: Lejeuneaceae) - a new record for Indian bryoflora from Lohit district, Arunachal Pradesh. Indian J. Forest. 32: 653-656.

Dey, M. and D.K. Singh. 2010a. Drepanolejeunea fleischeri (Steph.) Grolle \& R.L.Zhu (Hepaticae: Lejeuneaceae): an addition to Indian Himalaya. Geophytology 39: 65-71.

Dey, M. and D.K. Singh. 2010b. Two new epiphyllous Leptolejeunea (Hepaticae: Lejeuneaceae) from Eastern Himalaya, India. Taiwania 55: 355-362.

Dey, M. and D.K. Singh. 2011a. Four foliicolous species of Cololejeunea (Spruce) Schiffn. (Marchantiophyta: Lejeuneaceae) new to India. J. Bryol. 33: 163 - 167. doi: 10.1179/1743282010Y.0000000022

Dey, M. and D.K. Singh. 2011b. A new Lopholejeunea (Spruce) Schiffn. (Hepaticae: India. Nelumbo 53: 197-200.

Lejeuneaceae) from

Dey, M. and D.K. Singh. 2012. Epiphyllous liverworts of Eastern Himalaya. Botanical Survey of India, Kolkata. 364 pp.

Dey, M. and D.K. Singh. 2016a. Three foliicolous taxa of Lejeuneaceae (Marchantiophyta) new to India from Great Nicobar Biosphere Reserve, Andaman \& Nicobar Islands. Acta. Bot. Hung. 58 (1-2): 49-68. doi: 10.1556/034.58.2016.1-2.2

Dey, M. and D.K. Singh. 2016b. A new species and a new record of Cololejeunea (Lejeuneaceae, Marchantiophyta) from Andaman \& Nicobar Islands, India. Cryptog. Bryol. 37 (2): doi/10.7872/cryb/v37.iss2.2016.1

Dey, M., D. Singh and D.K. Singh. 2007. Lejeunea eifrigii Mizut. (Hepaticae: Lejeuneaceae) - a new record for Indian bryoflora from Sikkim. Indian J. Forest. 30: 511-512.

Dey, M., D. Singh and D.K. Singh. 2008a. A new species of Cololejeunea (Hepaticae: Lejeuneaceae) from Eastern Himalaya, India. Taiwania 53: 258-263. doi: 10.1179/174328208X300651

Dey, M., D. Singh and D.K. Singh. 2009. Some new and noteworthy records of Hepaticae from Eastern Himalaya, India. Indian J. Forest. 32: 669-684.

Dey, M., D. Singh and D.K. Singh. 2010. Two more additions to Indian Cololejeunea (Hepaticae: Lejeuneaceae) from West Sikkim. Indian J. Forest. 33: 615-618.
Dey, M., D. Singh and D.K. Singh. 2011. Jubula pennsylvanica (Hepaticae: Jubulaceae) new to Asia. Taiwania 56: 66-70.

Dey, M., D. Singh and D.K. Singh. 2013. The genus Drepanolejeunea (Marchantiophyta: Lejeuneaceae) in India with a note on three new additions from Eastern Himalaya Tropical Bryol. 35:14-25.

Dey, M., D.K. Singh and D. Singh. 2008b. Two new species of Lejeunea Lib. (Hepaticae: Lejeuneaceae) from Sikkim, India. J. Bryol. 30: 126-132.

Eshuo, K., A. Lokho, Adani and V. Doulo. 2013 Morphotaxonomic sudies on genus Radula Dumort. (Radulaceae: Hepaticae) from Nagaland, North East India. Indian J. Plant Sci. 2 (3): 66-72.

Gradstien, S.R. 2013. A classification of Lejeuneaceae (Marchantiophyta) based on molecular and morphological evidence. Phytotaxa 100: 6-20. doi: 10.11646/phytotaxa.100.1.2

Herzog, T. 1950. Miscellanea bryologica II. Palaeotropica. Mem. Soc. Fauna Flora Fenn. 26: 37-66.

Kachroo, P. 1951. Studies in Assam Hepaticae I. On some epiphyllous liverworts of Assam. J. Univ. Gauhati 2: 3139.

Kumar, S., T. Pócs. and S.K. Singh. 2015. Two Drepanolejeunea [Marchantiophyta] new to bryoftora of Meghalaya. Indian J. Forest. 38 (4): 343-349.

Lal, J. 1977. Colura Dum. (Hepaticae) - a genus new to Indian Flora. Curr. Sci. 46: 618.

Lal, J. 1979. Epiphyllous bryophytes of Manipur I. Metzgeriaceae. Proc Indian Sci .Congress 42-43.

Lal, J. 1980. Colura ari St. (Hepaticae) from Andamans - a new record for India. Bull. Bot. Surv. India 22: 207-209.

Lal, J. 2003. Studies in Epiphyllous liverworts of India - its present position. Bull. Bot. Surv. India 45: 179-186.

Manju, C.N., T. Pócs, K.P. Rajesh and R. Prakashkumar. 2012. Lejeuneaceae (Marchantiophyta) of the Western Ghats, India. Acta Biol. Plant Agriensis 2: 127-147.

Mittermeier, R.A., P.R. Gil and C.G. Mittermeier 1997. Megadiversity: Earth's Biologically Wealthiest Nations. Conservation International, Cemex.

Pandé, S.K. 1958. Some aspects of Indian Hepaticology (Presidential address). J. Indian Bot. Sco. 15: 221-233.

Pandé, S.K. and R.N. Misra. 1943. Studies in Indian Hepaticae II. On the epiphyllous liverworts of India and Ceylon I. J. Indian Bot. Soc. 22: 159-169.

Pandé, S.K., K.P. Srivastava and S. Ahmad. 1957. Epiphyllous liverworts of India and Ceylon - II. J. Indian Bot. Soc. 36: 335-347.

Singh, D. 2016. Two new records of family Lejeuneaceae (Marchantiophyta) from Sikkim, India. Indian J. Forest. (in press).

Singh, D. and D.K. Singh. 2008. A note on Metzgeria conjugata Lindb. (Hepaticae: Metzgeriaceae) from India. Geophytology 37: 47 - 49.

Singh D. and D.K. Singh. 2013. Some new and noteworthy records of family Lejeuneaceae (Marchantiophyta) from Sikkim, India. Nelumbo 55: 153-165.

Singh, D., M. Dey and D.K. Singh. 2007. Diversity in epiphyllous taxa of genus Metzgeria Raddi from Eastern Himalaya with a note on three new records of genus from India. Proc. Nat. Acad. Sci. India 77B: 375-386.

Singh, D., M. Dey and D.K. Singh. 2008. Lejeunea papilionacea Steph. (Hepaticae: Lejeuneaceae) an addition to Indian bryoflora from East Sikkim. Bull. Bot. Surv. India 50: 212-215.

Singh, D., M. Dey and D.K. Singh. 2010a. A Synoptic Flora of Liverworts and Hornworts of Manipur. Nelumbo 52: 9-52.

Singh, D., M. Dey and D.K. Singh. 2010c. Leptolejeunea apiculata (Horik.) S.Hatt. (Hepaticae: Lejeuneaceae) 
new to Indian bryoflora. Indian J. Forest. 33: 395398.

Singh, D., M. Dey and G.K. Upadhyay. 2010b. A preliminary survey of Hepaticae of Little Andaman Island. Nelumbo 52: 125-130.

Singh, D.K. 1992. Liverworts (Hepaticae) diversity in India and its conservation. In: Status Report of Biodiversity Conservation in India. Ministry of Environment and Forests, New Delhi. pp. 92.

Singh, D.K. 1996. Hepaticae (Bryophyta). In: P.K. Hajra (ed.) A contribution to the flora of Namdapha Arunachal Pradesh. Botanical Survey of India, New Delhi. pp. 46-67.

Singh, D.K. 1997. Liverworts. In: Mudgal, V. and P.K. Hajra (eds.), Floristic Diversity and Conservation Strategies in India. I. Botanical Survey of India, Dehradun. pp. 235-300.

Singh, D.K. and M. Dey. 2010. Genus Tuyamaella S.Hatt. (Lejeuneaceae) in India. Nelumbo 52: 135-137.

Singh, D.K., M. Dey and D. Singh. 2016. The genus Radula (Radulaceae, Marchantiophyta) in Andaman \& Nicobar Islands, India. J. Bryol.: doi 10.1179/1743282015Y.0000000032

Singh, D.K., S. Das and M. Dey. 2006b. Leptolejeunea subdentata Schiffn. ex Herzog (Hepaticae: Lejeuneaceae) New to India. Indian J. Forest. 29: 339-342.

Singh, D.K., S.K. Singh and M. Dey. 2006a. On a collection of Hepaticae from Andaman Islands. Phytotaxonomy 6: 99-104.

Singh, D.K., S. Majumdar and D. Singh. 2014. Cheilolejeunea osumiensis (Marchantiophyta: Lejeuneaceae) - an addition to the Indian bryoflora from Eastern Himalaya. Nelumbo 56: 259-264.

Singh, S.K. and H.A. Barbhuiya. 2012. A compendium to Marchantiophyta and Anthocerotophyta of Assam, India. Arch. Bryol. 149: 1-30.

Singh, S.K. and H.A. Barbhuiya. 2013. Contributions to the Hepaticae and Anthocerotae of Mizoram VI. Cololejeunea chenii new to India. Acta. Bot. Hung. 55: 135-139. doi: 10.1556/ABot.55.2013.1-2.9

Singh, S.K. and M. Dey. 2012. A new species of Drepanolejeunea (Marchantiophyta: Lejeuneaceae) from India. Nelumbo 54: 20-23.

Singh, S.K. and M. Dey. 2014. Two noteworthy species of Cololejeunea (Marchantiophyta: Lejeuneaceae) from Mizoram. Nelumbo 56: 273-277.

Singh, S.K. and T. Pócs. 2016. Reinstatement of Cololejeunea indica Pandé \& Misra, a name previously synonymous with $C$. spinosa (Horik.) Pandé \& Misra. Cryptog. Bryol. 37 (1): 27-32.

Singh, S.K., T. Pócs and S. Kumar. 2015. Notes on four new and noteworthy records of Lejeunea from Meghalaya, India. Acta. Bot. Hung. 57 (3-4): 407418. doi: 10.1556/034.57.2015.3-4.10
Sreebha, R., K.C. Kariyappa and A.E.D. Daniels. 2015. Notes on a little known species of Cololejeunea (Marchantiophyta) recently collected in the Western Ghats of Tamil Nadu. Nelumbo 57: 92-95.

Srivastava, K.P. 1960. Studies in Indian Metzgerineae - IV. Riccardia levieri Schiffn. J. Indian Bot. Soc. 39: 537-547.

Srivastava, S.C. and G. Srivastava. 1989a. Two Cololejeunea from south India. Proc. Indian Acad. Sci., Pl. Sci. 99: 83-90.

Srivastava, G. and S.C. Srivastava.1989b. Present status of Cololejeunea subgenus Cololejeunea in India. Geophytology 19: 120-130.

Swartz, O. 1788. Nova Genera \& Species Plantarum seu Prodromus. Bibliopoliis Acad. M. Swederi, Upsaliae.

Udar, R. and D. Kumar. 1984. Epiphyllous taxa of Radula Dumort. from India. Geophytology 14: 161-170.

Udar, R. and G. Srivastava. 1982. Two new species of Cololejeunea from Peninsular India. J. Bryol. 12: 227- 231.

Udar, R. and G. Srivastava. 1983. A new Cololejeunea from India. Misc. Bryol. Lichenol. 9: 137-139.

Udar, R. and G. Srivastava. 1985. Cololejeunea (Pedinolejeunea) kashyapii sp. nov. from Karnataka, India. Geophytology 15: 64-66.

Udar, R. and U.S. Awasthi. 1982. Leptolejeunea foliicola St. - new to Indian Bryoflora. J. Indian Bot. Soc. 61: 299-301.

Udar, R. and U.S. Awasthi. 1984. The genus Rhaphidolejeunea Hork. in India. Yushania 1: 15-17.

Udar, R. and U.S. Awasthi. 1985. Colura acroloba (Mont.) JovetAst. - new to Indian Bryoflora. J. Indian Bot. Soc. 64: 284286.

Udar, R., G. Srivastava and S.C. Srivastava. 1985. Cololejeunea (Pedinolejeunea) furcilobulata (Berrie et Jones) Schuster: new to Asia. Proc .Indian Acad. Sci., Pl. Sci. 95: 303-307.

Verma, P.K. and S.C. Srivastava. 2008. Diversity and distribution of epiphytic liverworts (Hepaticae) in Nilgiri hills (Tamil Nadu). Proc. Nat. Acad. Sci. India 78B: 253-264.

Verma, P.K., A. Alam and K.K. Rawat. 2013. Assessment of liverwort and hornwort flora of Nilgiri Hills, Western Ghats (India). Polish Bot. Journal 58 (2): 525-537. doi: 10.2478/pbj-2013-0038

Wang, J, S.R. Gradstein, X.-Q. Shi and R.-L. Zhu. 2014. Phylogenetic position of Trocholejeunea and a new infrageneric classification of Acrolejeunea (Lejeuneaceae, Marchantiophyta). Bry. Div. Evo. 36 (1): 31-44. doi: 10.11646/bde.36.1.3

Williams, J., C. Read, A. Norton, S. Dovers, M. Burgman, W. Proctor and H. Anderson. 2001. Biodiversity, Australia State of the Environment Report 2001 (Theme Report). CSIRO, Canberra.

Zhu, R.-L. and D.G. Long. 2003. Lejeuneaceae (Hepaticae) from several recent collections from the Himalaya. J. Hattori Bot. Lab. 93: 101-115.

Zhu, R.-L. and M.L. So. 2001. Epiphyllous liverworts of China. Beih. Nova Hedwigia 121: 1-418. 